\title{
ELEMENTARY DUALITY OF MODULES
}

\author{
IVO HERZOG
}

\begin{abstract}
Let $R$ be a ring. A formula $\varphi(\mathbf{x})$ in the language of left $R$-modules is called a positive primitive formula (ppf) if it is of the form $\exists \mathbf{y}(A B)\left(\begin{array}{l}\mathbf{x} \\ \mathbf{y}\end{array}\right)=\mathbf{0}$ where $A$ and $B$ are matrices of appropriate size with entries in $R$. We apply Prest's notion of $D \varphi(\mathbf{x})$, the ppf in the language of right $R$-modules dual to $\varphi$, to show that the model theory of left $R$-modules as developed by Ziegler [Z] is in some sense dual to the model theory of right $R$-modules. We prove that the topologies on the left and right Ziegler spectra are "isomorphic" (Proposition 4.4). When the lattice of ppfs is well behaved, there is a homeomorphism $D$ between the left and right $Z$ iegler spectra which assigns to a given pure-injective indecomposable left $R$-module $U$ the dual pure-injective indecomposable right $R$-module $D U$. Theorem 6.6 asserts that given a complete theory $T$ of left $R$-modules, there is a dual complete theory $D T$ of right $R$-modules with corresponding Baur-Garavaglia-Monk invariants. In the end, we give some conditions on a pure-injective indecomposable ${ }_{R} U$ which ensure that its dual $D U$ may be represented as a hom set of the form $\operatorname{Hom}_{S}\left({ }_{R} U_{S}, E_{S}\right)$ where $S$ is some ring making ${ }_{R} U_{S}$ into a bimodule and $E_{S}$ is injective.
\end{abstract}

Prest [P, Chapter 8] defined the dual of a positive-primitive formula $\varphi(\mathbf{x})$ of the form $\exists \mathbf{w}\left(H_{1} H_{2}\right)\left(\begin{array}{l}\mathbf{x} \\ \mathbf{w}\end{array}\right)=0$ to be $(D \varphi)(\mathbf{x}) \Leftrightarrow \exists \mathbf{v}(\mathbf{x v})\left(\begin{array}{cc}I & 0 \\ H_{1} & H_{2}\end{array}\right)=0$ (Definition 2.1). In this paper, we develop an elementary theory of duality for modules by extending Prest's definition to an appropriate category of functors. From this and the work of Ziegler [ $\mathrm{Z}]$ arises a reflexive class of pure-injective indecomposable modules.

In $\S 1$, we define $(R \text {-Mod) })^{\text {eq }}$, a category of functors from $R$-Mod, the category of left $R$-modules, to $\mathrm{Ab}$, the category of abelian groups. $(R \text {-Mod) })^{\text {eq }}$ is an abelian reduct of Shelah's $L^{\text {eq }}[P, \S 10 . T]$ which has proven sufficient for most purposes. We define (Mod- $R)^{\mathrm{eq}}$, the right analogue of $(R \text {-Mod })^{\mathrm{eq}}$, similarly and the content of Theorem 2.9 is that these two categories are isomorphic.

Nearly all our proofs depend on the following paraphrase of [S, Proposition I.8.8].

Proposition 3.2. If $\mathbf{a} \in N_{R}$ and $\mathbf{c} \in{ }_{R} M$ are tuples of the same length, then $\mathbf{a} \otimes \mathbf{c}=0$ in $N \otimes_{R} M$ iff there is a ppf $\varphi(\mathbf{x}) \in_{R} L$ such that ${ }_{R} M \vDash \varphi(\mathbf{c})$ and $N_{R} \vDash(D \varphi)(\mathbf{a})$.

Received by the editors March 5, 1990 and, in revised form, February 12, 1991.

1980 Mathematics Subject Classification (1985 Revision). Primary 16A64; 16A49; Secondary $03 \mathrm{C} 60$.

This article forms a part of the author's doctoral dissertation, written at the University of Notre Dame under the supervision of Anand Pillay. 
As a consequence, the definition of tensoring may be extended to the objects of $(R \text {-Mod })^{\mathrm{eq}}$.

Ziegler [Z, Theorem 4.9] noticed that the set of (isomorphism types of) left (right) pure-injective indecomposables may be endowed with a topology and that a basis for this topology may be taken as the set of supports of the objects of $(R \text {-Mod })^{\text {eq }}\left((\operatorname{Mod}-R)^{\mathrm{eq}}\right)$. The duality between $(R \text {-Mod })^{\text {eq }}$ and (Mod- $\left.R\right)^{\text {eq }}$ shows that the left and right Ziegler spectra have the same topology (Proposition 4.4). This leads to the definition of a reflexive pure-injective indecomposable $U$ and its dual $D U$ whose lattices of pp-definable subgroups are related by

Theorem 4.10. Duality, the map which takes $U$ to $D U$, is a bijection from the set of (isomorphism types of) reflexive pure-injective indecomposable left $R$ modules to the set of (isomorphism types of) reflexive pure-injective indecomposable right $R$-modules and conversely. For a reflexive $U$, we have

(i) $D D U=U$,

(ii) $\operatorname{Latt}(D U) \cong \operatorname{Latt}(U)^{\text {op }}$ via the map which takes $\varphi(U)$ to $D \varphi(D U)=$ $\{\mathbf{c} \in D U \mid \mathbf{c} \otimes \varphi(U)=0$ in $D U \otimes U\}$.

As an example, the dual of a pure-injective indecomposable module over a Dedekind domain is determined in $\S 5$.

Prest's notion of localization [P, Chapter 8$]$ is then applied to our previous results and one can define the dual $D T$ of a complete theory $T$ of left (right) $R$-modules. This allows us to show that, model-theoretically, left and right $R$-modules are intimately related.

Theorem 6.6. Duality, the map which takes $T$ to $D T$, is a bijection from the class of complete theories of left (right) $R$-modules to the class of complete theories of right (left) $R$-modules. $D T$ is uniquely determined by $T$ via the equation $|\varphi / \psi|_{T}=|D \psi / D \varphi|_{D T}$. If $U$ is reflexive, then $D(\operatorname{Th}(U))=\operatorname{Th}(D U)$.

As an application of Theorem 6.6, the dual of an indecomposable strongly minimal module may be endowed with a topology which makes it a compact (Hausdorff) topological module (see $\S 7$ ).

Our shift of emphasis (from finitely presented modules) to the pure-injective indecomposables is justified by

Proposition 8.1. ${ }_{R} N \stackrel{f}{\longrightarrow}{ }_{R} M$ is pure iff $U \otimes_{R} N \stackrel{1 \otimes f}{\longrightarrow} U \otimes_{R} M$ is injective for each pure-injective indecomposable right $R$-module $U$.

This gives rise to the notion of local purity and the result (Proposition 8.9) that $D U$ is a direct summand of $\operatorname{Hom}(U, D U \otimes U)$.

As an application of $\S 8$, we prove that the dual of a flat (pure-injective) module is injective and we put such findings in the context of the work of Eklof and Sabbagh [E-S, S-E] on (left) coherent rings. We also prove

Theorem 10.5. If ${ }_{R} U$ is a totally transcendental indecomposable $R$-module and $S=\operatorname{End}_{R} U$, then $D U_{R} \cong \operatorname{Hom}_{S}\left({ }_{R} U_{S}, D U \otimes U_{S}\right)$.

Theorem 11.2. If $U$ is a definably finitely generated totally transcendental indecomposable module, then $D U \otimes U_{S}=E\left(\Delta_{S}\right)$, where $S=\operatorname{End}_{R} U, \Delta$ is its residue ring and $E\left(\Delta_{S}\right)$ is the injective hull of $\Delta_{S}$, 
I thank Edgar Enochs and Željko Sokolovič for some very helpful conversations.

\section{Preliminaries}

Although this article is not meant to be self-contained, we shall set down in this section some of the necessary definitions and notation. We also take this opportunity to emphasize certain aspects of the basic notions which are usually left implicit, but will be of great use to us in the sequel. The reader is referred to $[\mathrm{P}]$ or $[\mathrm{Z}]$ for a thorough treatment of what follows. Indeed, both of these references are comprehensive enough that we shall routinely cite them in favor of the original source.

We use left subscripts to indicate that left $R$-modules are in question and a right subscript to indicate the same for right $R$-modules. The symbol $\Leftrightarrow$ will be used to express equality of formulae (as opposed to mere equivalence) and tuples a will be printed boldface, while single elements $a$ are written plainly. $l(\mathbf{a})$ is the length of $\mathbf{a}$.

The language ${ }_{R} L=(+,-, 0, r)_{r \in R}$ used for the study of left $R$-modules consists of the binary function symbol + , the unary function symbol - , a consiant symbol 0 , and for each $r \in R$ a unary function symbol, also denoted by $r$. A similar language $L_{R}=(+,-, 0, r)_{r \in R}$ is used for studying right $R$ modules. In this latter case, however, each $r \in R$ interprets a unary function which acts on its argument from the right. $1 \in R$ and all modules are unitary.

\section{A. Positive primitive formulae.}

Definition 0.1. A positive primitive formula (henceforth ppf) of ${ }_{R} L$ is one of the form $\varphi(\mathbf{x}) \Leftrightarrow \exists \mathbf{y} A \mathbf{x}+B \mathbf{y}=0$ where $A$ and $B$ are matrices with entries in $R$ and $A \mathbf{x}$ e.g. really means $A \mathbf{x}^{t}$. A ppf in $L_{R}$ has the similar form $\varphi(\mathbf{x}) \Leftrightarrow$ $\exists \mathbf{y} \mathbf{x} A+\mathbf{y} B=0$. Unless it is said otherwise, $\varphi, \psi, \sigma, \tau, \rho, \delta$ and $\varepsilon$ will always denote ppfs. If $\varphi(x)$ is a ppf in, say, one variable, then

$$
\varphi^{n}(\mathbf{x}) \Leftrightarrow \bigwedge\left\{\varphi\left(x_{i}\right) \mid 0 \leq i<n\right\} \quad \text { where } l(\mathbf{x})=n .
$$

Given ${ }_{R} M \in R$-Mod and $\varphi(\mathbf{x})$, then $\varphi(M)=\left\{\mathbf{a} \in M^{n} \mid M \vDash \varphi(\mathbf{a})\right\}$ where $n=l(\mathbf{a})=l(\mathbf{x})$. This is a subgroup of $\left(M^{n},+\right)$ and all such subgroups will be referred to as a pp-definable. The mapping which assigns to $M$ the ppdefinable subgroup $\varphi(M)$ constitutes a functor from $R$-Mod to Ab if for each $R$-homomorphism $f: M \rightarrow N$ we set $\varphi(f)$ to be the restriction of $f$ to $\varphi(M)$. Note that the functor $\varphi$ commutes with direct sums. The same considerations hold for right $R$-modules.

The pp-definable subgroups of $M$ form a lattice under the operations $\cap$ and + and if $\varphi(M)$ and $\psi(M)$ are such subgroups (in the same number of variables) we denote by $\varphi+\psi$ the ppf $\exists \mathbf{y}, \mathbf{z}|\varphi(\mathbf{y}) \wedge \psi(\mathbf{z}) \wedge \mathbf{x}=\mathbf{y}+\mathbf{z}|$ which defines the subgroup $\varphi(M)+\psi(M)$ and by $\varphi \wedge \psi$ the ppf which defines the subgroup $\varphi(M) \cap \psi(M)$. The prominence which ppfs enjoy in the model theory of modules stems from the fact due to Baur [P, Corollary 2.16; Z, Theorem 1.1] that modulo a complete theory $T$, every formula is equivalent to a boolean combination of ppfs. We shall not have recourse to this result directly, only because it has been so completely absorbed into the subject. 
A pp-pair $\varphi / \psi$ is just a pair of ppfs $\varphi$ and $\psi$ (in the same number of variables) such that $\vDash \psi \rightarrow \varphi$. If it ever happens that we mention a pair $\sigma / \tau$ where it is not clear that $\vDash \tau \rightarrow \sigma$, it is because we really mean $\sigma / \sigma \wedge \tau$. As above, each pp-pair $\varphi / \psi$ determines a functor from $R$-Mod (Mod- $R$ ) to Ab, the quotient of the functor $\varphi$ by the functor $\psi$. So $\varphi / \psi(M)=\varphi(M) / \psi(M)$ and for $f \in \operatorname{Mor}_{R}(M, N), \varphi / \psi(f): \varphi / \psi(M) \rightarrow \varphi / \psi(N)$ is just the induced Abmorphism. The importance of pp-pairs lies in the fact [P, Corollary 2.18] that two modules $M$ and $N$ are elementarily equivalent iff $|\varphi / \psi(M) \vDash| \varphi / \psi(N) \mid$ $(\bmod \infty)$ for each pair of ppfs in one variable $(\kappa=\lambda(\bmod \infty)$ if $\kappa=\lambda$ or both are infinite).

Definition 0.2. For a complete theory $T$ and a pp-pair $\varphi / \psi,|\varphi / \psi|_{T} \in \omega \cup\{\infty\}$ is defined as $|\varphi / \psi|_{T}=\sup \{n|T \vDash| \varphi / \psi \mid \geq n\}$ (where $n<\infty$ for each $n \in \omega$ ).

The $|\varphi / \psi|_{T}$ are called the BGM-invariants (Baur-Garavaglia-Monk) of a theory $T$ and according to the above, they determine $T$.

If $\mathbf{a} \in M$, then $\operatorname{tp}^{+}(\mathbf{a})=\{\varphi(\mathbf{x}) \mid M \vDash \varphi(\mathbf{a})\}$ and $\left.\operatorname{tp}^{-} \mathbf{a}\right)=\{\psi(\mathbf{x}) \mid M \vDash$ $\neg \psi(\mathbf{a})\}$. Then pp-tp(a) $=\operatorname{tp}^{+}(\mathbf{a}) \cup\left\{\neg \psi(\mathbf{x}) \mid \psi(\mathbf{x}) \in \operatorname{tp}^{-}(\mathbf{a})\right\}$ is the pp-type of a . Conversely, a pp-type $p(\mathbf{x})$ is a maximal consistent (with some theory of $R$-modules) set of ppfs and their negations. $p^{+}(\mathbf{x})=\{\varphi(\mathbf{x}) \mid \varphi(\mathbf{x}) \in p(\mathbf{x})\}$ is the positive part of $p$ and $p^{-}(\mathbf{x})=\{\psi(\mathbf{x}) \mid \neg \psi(\mathbf{x}) \in p(\mathbf{x})\}$ is the negative part of $p$. If $p$ is a pp-cype and $\varphi / \psi$ is a pp-pair, we say $\varphi / \psi \in p$ to indicate that $\varphi \in p^{+}$and $\psi \in p^{-}$.

B. Purity. A homomorphism $f:{ }_{R} M \rightarrow{ }_{R} N$ is pure if one of the conditions of the next proposition hold.

Proposition 0.3 [S, §I.11]. The following are equivalent:

(i) For each $p p f \varphi(\mathbf{x}), N \vDash \varphi(f \mathbf{a})$ implies $M \vDash \varphi(\mathbf{a})$.

(ii) $K_{R} \otimes M \rightarrow K_{R} \otimes N$ is injective for each $K \in \operatorname{Mod}-R$.

(iii) As (ii), but with $K$ finitely presented.

Note that $f$ is pure iff for each pp-pair $\varphi / \psi, \varphi / \psi(f)$ is an injection. So, in particular, pure homomorphisms are imbeddings.

A module $M$ is pure injective if every pure imbedding of $M$ splits. Thus a homomorphism from a pure submodule of $N$ to $M$ lifts to a homomorphism from $N$ to $M$. If $A \subseteq M$ is just a subset, then $H_{M}(A)$, a pure injective hull of $A$ in $M$ is nothing more than a minimal direct summand of $M$ containing $A$. It always exists and it is unique up to $A$-isomorphism [P, Theorem 4.10, $\mathrm{Z}$, Theorem 3.6]. Indeed, the isomorphism type of $H_{M}(A)$ depends only on the $\operatorname{pp}-\operatorname{tp}(A)$ and so it makes sense to speak about $H(p)$, the hull of the pptype $p(\mathbf{x})$. If we have the occasion to mention $H(A)$ without there being any pure-injectives in sight, it is because we always assume that we live in a huge, sufficiently saturated (and hence pure-injective) model of ${ }_{R} T\left(T_{R}\right)$ (see below). For our ends, the following characterization of pure-injectives will be the most useful.

Proposition 0.4 [P, Theorem 2.8]. $M$ is pure-injective iff every type $p^{+}(\mathrm{x} / A)$ of ppfs with parameters in $A \subseteq M$ which is finitely satisfiable in $M$, has a realization $\mathbf{c} \in M$.

A pure-injective module is indecomposable iff it has a local endomorphism ring [Z, Theorem 4.3]. A pp-type $p(\mathbf{x})$ is indecomposable if $H(p)$ is. 
C. The Ziegler spectrum. Ziegler noticed [Z, Theorem 4.9] that ${ }_{R} I\left(I_{R}\right)$, the space of isomorphism types of pure-injective indecomposable left (right) $R$ modules may be endowed with a topology in which the support of the functor $\varphi / \psi,(\varphi / \psi)=\left\{U \in{ }_{R} I\left(I_{R}\right) \mid \varphi / \psi(U) \neq 0\right\}$, is an open set. In fact, we may take $\{(\varphi / \psi) \mid \varphi / \psi$ is a pp-pair of ppfs in one variable $\}$ as a basis for Ziegler's topology. We shall adhere to the convention that $O$ denotes an open subset of ${ }_{R} I\left(I_{R}\right)$ and $I \subseteq{ }_{R} I\left(I_{R}\right)$ a closed set.

Given a complete theory $T$, we may associate to $T$ the closed set $I(T)=$ $\left\{U \in{ }_{R} I\left(I_{R}\right) \mid U\right.$ occurs as a direct summand of some model of $\left.T\right\}$ and given a closed set $I$, there is a complete theory $T$ for which $I=I(T)$ (just let $T=\operatorname{Th}(\bigoplus\{U \mid U \in I\}))$ [Z, Corollary 4.10]. There is a unique complete theory $T(I)$ closed under products for which $I=I(T(I))$ and if $I=I(T)$, then $T^{\omega}=T(I)$. We abbreviate $T\left({ }_{R} I\right)\left(T\left(I_{R}\right)\right)$ by ${ }_{R} T\left(T_{R}\right)$. For a module $N$, set $I(N)=I(\operatorname{Th}(N))$. If $U \in{ }_{R} I\left(I_{R}\right)$, then $I(U)$ is the closure of $\{U\}$ in ${ }_{R} I\left(I_{R}\right)$.

The lattice of ppfs of a complete theory $T=\operatorname{Th}(N), \operatorname{Latt}(T)$, is defined as a lattice isomorphic to $\operatorname{Latt}(N)$, the lattice of pp-definable subgroups of $N$. This determines $\operatorname{Latt}(T)$ within isomorphism and does not depend on the particular model chosen. To be precise, we have for each $n<\omega$, $\operatorname{Latt}_{n}(T)$, the lattice of ppfs in $n$ variables, but whatever we prove about $\operatorname{Latt}_{n}(T)$ will hold for arbitrary $n$ so we omit the subscript. The elements of $\operatorname{Latt}(T)$ are represented by $\operatorname{ppfs} \varphi$. If $I=I(T)=I\left(T^{\prime}\right)$, then $\operatorname{Latt}(T)=\operatorname{Latt}\left(T^{\prime}\right)$ and so we can associate to $I$ a pp-lattice $\operatorname{Latt}(I)=\operatorname{Latt}(T)$. We endow $I$ with a dual nature. Along with being a closed set, I will also be the (not necessarily complete) theory given in

Definition 0.5. $I=\{\varphi(\mathbf{x}) \rightarrow \psi(\mathbf{x}) \mid \varphi \leq \psi$ in $\operatorname{Latt}(I)\} \cup\{\neg(\varphi(\mathbf{x}) \rightarrow \psi(\mathbf{x})) \mid$ $\neg(\varphi \leq \psi)$ in $\operatorname{Latt}(I)\}$.

Note that $I \cap(\varphi / \psi)=\varnothing$ iff $\varphi \leq \psi$ in Latt $(I)$. We can think of $I$ as a set of formulae of the form $|\varphi / \psi|>1$ or $|\varphi / \psi|=1$ so that $I$ can distinguish between trivial $(=1)$ and nontrivial $(>1)$ BGM-invariants, but it does not know their cardinalities. The BGM-invariants of $T(I)$ are given as

$$
|\varphi / \psi|_{T(I)}= \begin{cases}\infty & \text { if } I \vDash|\varphi / \psi|>1, \\ 1 & \text { if } I \vDash \mid \varphi / \psi \vDash 1 .\end{cases}
$$

For example ${ }_{R} I\left(I_{R}\right) \vDash|\varphi / \psi|=1 \quad \varphi(M) \subseteq \psi(M)$ if for every left (right) $R$ module $M$, which we shall write as ${ }_{R} I\left(I_{R}\right) \vDash \varphi(\mathbf{x}) \rightarrow \psi(\mathbf{x})$. It will always be clear from the context whether we are considering $I$ as a closed set or a theory.

There is a measure of complexity, $m$-dimension, that one may place on Latt $(I)$ which indicates how well behaved $I$ is.

Definition 0.6. For a pp-pair $\varphi / \psi, m-\operatorname{dim}_{I}(\varphi / \psi) \geq \alpha$ is defined by recursion on the ordinal $\alpha$ as follows.

(i) $m-\operatorname{dim}_{I}(\varphi / \psi) \geq 0$ if $I \vDash|\varphi / \psi|>1$.

(ii) If $\lambda$ is a limit ordinal, then $m$ - $\operatorname{dim}_{I}(\varphi / \psi) \geq \lambda$ if $m$ - $\operatorname{dim}_{I}(\varphi / \psi) \geq \gamma$ for all $\gamma<\lambda$.

(iii) $m$ - $\operatorname{dim}_{I}(\varphi / \psi) \geq \alpha+1$ if there is an infinite descending chain $\varphi=\varphi_{0} \supseteq$ $\varphi_{1} \supseteq \varphi_{2} \supseteq \cdots \supseteq \psi$ such that $m-\operatorname{dim}_{I}\left(\varphi_{n} / \varphi_{n+1}\right) \geq \alpha$ for each $n<\omega$ or if there is an infinite ascending chain $\psi=\psi_{0} \subseteq \psi_{1} \subseteq \psi_{2} \subseteq \cdots \subseteq \varphi$ such that $m-\operatorname{dim}_{I}\left(\psi_{n+1} / \psi_{n}\right) \geq \alpha$ for each $n<\omega$. 
We set $m-\operatorname{dim}_{I}(\varphi / \psi)=\alpha$ if $m-\operatorname{dim}_{I}(\varphi / \psi) \geq \alpha$ but not $m-\operatorname{dim}_{I}(\varphi / \psi) \geq$ $\alpha+1$. If $m$-dim $\operatorname{dim}_{I}(\varphi / \psi) \geq \alpha$ for every ordinal $\alpha$, we say $m$-dim $\operatorname{dim}_{I}(\varphi / \psi)=\infty$ and we say $m$-dim $I(\varphi / \psi)<\infty$ otherwise. $m$-dim $(I)$ is defined as

$$
m-\operatorname{dim}_{I}(x=x / x=0) .
$$

If, for example, $m$ - $\operatorname{dim}(I(M))=0$, then $M$ has a pp-composition series. In [Z. Theorem 8.6], Ziegler expressed the intimate relationship between the CantorBendixson rank of the closed set $I$ and $\operatorname{Latt}(I)$. The following is a special case.

Proposition 0.7. If $m-\operatorname{dim}(I)<\infty$, then for each pp-pair $\varphi / \psi, m-\operatorname{dim}_{I}(\varphi / \psi)=$ CB-rk $(I \cap(\varphi / \psi))$, the Cantor-Bendixson rank of $I \cap(\varphi / \psi)$.

\section{The CATEgory $(R-\mathrm{Mod})^{\mathrm{eq}}$}

In this section, we make an abelian category out of the functors $\varphi / \psi$. The morphisms will be the pp-definable natural transformations. We call this category $(R \text {-Mod })^{\mathrm{eq}}$. Let us define $(R \text {-Mod })^{\mathrm{eq}}$.

a. $\mathrm{Ob}\left((R \text {-Mod })^{\mathrm{eq}}\right)$, the objects of $(R \text {-Mod })^{\mathrm{eq}}$, are the functors $\{\varphi / \psi \mid \varphi / \psi$ is a pp-pair in $\left.{ }_{R} L\right\}$. We mean, by this, that if ${ }_{R} I \vDash \varphi \leftrightarrow \varphi^{\prime}$ and ${ }_{R} I \vDash \psi \leftrightarrow$ $\psi^{\prime}$, then $\varphi / \psi$ and $\varphi^{\prime} / \psi^{\prime}$ are identified, as they represent the same functor. Sometimes we shall refer to the objects of $(R \text {-Mod })^{\text {eq }}$ as sorts.

b. $\rho \in \operatorname{Mor}(\varphi / \psi, \sigma / \tau)$ iff there is a ppf $\rho(\mathbf{x}, \mathbf{y}) \in{ }_{R} L$ such that

(i) ${ }_{R} I \vDash \varphi(\mathbf{x}) \rightarrow \exists \mathbf{y} \rho(\mathbf{x}, \mathbf{y})$,

(ii) ${ }_{R} I \vDash \psi(\mathbf{x}) \rightarrow \rho(\mathbf{x}, 0)$,

(iii) ${ }_{R} I \vDash \exists \mathbf{x} \rho(\mathbf{x}, \mathbf{y}) \rightarrow \sigma(\mathbf{y})$,

(iv) ${ }_{R} I \vDash \rho(0, \mathbf{y}) \rightarrow \tau(\mathbf{y})$.

This makes sense, for if $M \in R$-Mod and $\rho(\mathbf{x}, \mathbf{y})$ is a ppf as above, then we can define a morphism of abelian groups $\rho_{M}: \varphi(M) / \psi(M) \rightarrow \sigma(M) / \tau(M)$ as follows. For $\mathbf{a} \in \varphi(M), \rho_{M}(\mathbf{a}+\psi(M))=\mathbf{b}+\tau(M)$ for some $\mathbf{b} \in M$ for which $M \vDash \rho(\mathbf{a}, \mathbf{b})$. (i) assures us that such a b exists, (iii) guarantees that $\mathbf{b} \in \sigma(M)$ and (iv) implies that $\mathbf{b}$ is unique modulo $\tau(M)$. (ii) says that $\psi(M)$ is contained in the kernel of this map and so $\rho_{M}$ is just the homomorphism induced on $\varphi(M) / \psi(M)$. We say that $\rho$ is represented by the ppf $\rho(\mathbf{x}, \mathbf{y})$ and, as for objects, we identify elements of $\operatorname{Mor}(\varphi / \psi, \sigma / \tau)$ which are represented by ${ }_{R} I$-equivalent ppfs.

Note that we have not restricted ourselves to $\mathrm{ppfs}$ in one variable. If, for example, $\varphi$ and $\psi$ are ppfs in $m$ variables and $\sigma$ and $\tau$ are ppfs in $n$ variables, then a ppf $\rho(\mathbf{x}, \mathbf{y})$ representing $\rho \in \operatorname{Mor}(\varphi / \psi, \sigma / \tau)$ is a ppf in $m+n$ variables, $l(\mathbf{x})=m, l(\mathbf{y})=n$.

Given $\rho_{1} \in \operatorname{Mor}(\varphi / \psi, \sigma / \tau)$ and $\rho_{2} \in \operatorname{Mor}(\sigma / \tau, \varepsilon / \delta)$ and representing ppfs $\rho_{1}(\mathbf{x}, \mathbf{y})$ and $\rho_{2}(\mathbf{y}, \mathbf{z})$, it is easy to check that the ppf (or one equivalent to it) $\rho_{2} \rho_{1}(\mathbf{x}, \mathbf{z}) \Leftrightarrow \exists \mathbf{y} \rho_{1}(\mathbf{x}, \mathbf{y}) \wedge \rho_{2}(\mathbf{y}, \mathbf{z})$ represents the natural transformation in $\operatorname{Mor}(\varphi / \psi, \varepsilon / \delta)$ obtained by composing $\rho_{1}$ with $\rho_{2}$.

The definition of (Mod- $R)^{\text {eq }}$, the corresponding category for right $R$-modules, is similar, with all formulae involved coming from $L_{R}$. But by this, we mean that $\rho \in \operatorname{Mor}(\psi / \varphi, \tau / \sigma)$ is a natural transformation from the functor $\tau / \sigma$ to the functor $\psi / \varphi$. In other words, $\rho$ acts on the right when right $R$-modules 
are in question. Thus in (Mod- $R)^{\text {eq }}$ :

$\mathbf{b}^{\prime} . \rho \in \operatorname{Mor}(\psi / \varphi, \tau / \sigma)$ iff there is a ppf $\rho(\mathbf{x}, \mathbf{y}) \in L_{R}$ such that

(i') $I_{R} \vDash \tau(\mathbf{y}) \rightarrow \exists \mathbf{x} \rho(\mathbf{x}, \mathbf{y})$,

(ii') $I_{R} \vDash \sigma(\mathbf{y}) \rightarrow \rho(0, \mathbf{y})$,

(iii') $I_{R} \vDash \exists \mathbf{y} \rho(\mathbf{x}, \mathbf{y}) \rightarrow \psi(\mathbf{x})$,

(iv') $I_{R} \vDash \rho(\mathbf{x}, 0) \rightarrow \varphi(\mathbf{x})$.

For $\rho_{1} \in \operatorname{Mor}(\psi / \varphi, \tau / \sigma)$ and $\rho_{2} \in \operatorname{Mor}(\tau / \sigma, \delta / \varepsilon)$, the composition $\rho_{2} \rho_{1}$ is represented by $\exists \mathbf{y} \rho_{1}(\mathbf{x}, \mathbf{y}) \wedge \rho_{2}(\mathbf{y}, \mathbf{z})$.

All this said, we are given an opportune moment to state

Goursat's Theorem. The morphism

$$
\rho \in \operatorname{Mor}(\exists \mathbf{y} \rho(\mathbf{x}, \mathbf{y}) / \rho(\mathbf{x}, 0), \exists \mathbf{x} \rho(\mathbf{x}, \mathbf{y}) / \rho(0, \mathbf{y})),
$$

represented by the ppf $\rho(\mathbf{x}, \mathbf{y})$, is an isomorphism.

\section{Duality}

Mike Prest noticed that to each ppf $\varphi(\mathbf{x}) \in{ }_{R} L$, there corresponds a dual ppf $D \varphi(\mathbf{x}) \in L_{R}$ and conversely. We shall extend the definition of $D$ to the objects and morphisms of $(R \text {-Mod })^{\text {eq }}$ in order to prove that $(R \text {-Mod })^{\text {eq }}$ and $(\operatorname{Mod}-R)^{\text {eq }}$ are isomorphic categories.

A. The dual of $\varphi(\mathbf{x})$. We shall begin with a brief mention of things contained in [P, Chapter 8].

Definition 2.1. (i) For a ppf $\varphi(\mathbf{x}) \in{ }_{R} L$ with $\varphi(\mathbf{x}) \Leftrightarrow \exists \mathbf{w}\left(\begin{array}{ll}H_{1} & H_{2}\end{array}\right)\left(\begin{array}{l}\mathbf{x} \\ \mathbf{w}\end{array}\right)=0$, define $D \varphi(\mathbf{x}) \in L_{R}$ as $D \varphi(\mathbf{x}) \Leftrightarrow \exists \mathbf{v}(\mathbf{x v})\left(\begin{array}{cc}I & 0 \\ H_{1} & H_{2}\end{array}\right)=0$ where $I$ is the $n \times n$ identity matrix, $n=l(\mathbf{x})=$ the number of columns in $H_{1}$ and $l(\mathbf{v})=$ the number of rows in $\left(H_{1} H_{2}\right)$.

(ii) Dually, if $\psi(\mathbf{x}) \in L_{R}$ with $\psi(\mathbf{x}) \Leftrightarrow \exists \mathbf{v}(\mathbf{x v})\left(\begin{array}{l}H_{1} \\ H_{2}\end{array}\right)=0$, then let $D \psi(\mathbf{x}) \in{ }_{R} L$ be the ppf $\exists \mathbf{x}\left(\begin{array}{ll}I & H_{1} \\ 0 & H_{2}\end{array}\right)\left(\begin{array}{l}\mathbf{x} \\ \mathbf{w}\end{array}\right)=0$.

That $D$ behaves well is the content of the following.

Proposition 2.2. (i) ${ }_{R} I\left(I_{R}\right) \vDash D^{2} \varphi(\mathbf{x}) \leftrightarrow \varphi(\mathbf{x})$ for each ppf $\varphi(\mathbf{x}) \in{ }_{R} L\left(L_{R}\right)$.

(ii) If ${ }_{R} I\left(I_{R}\right) \vDash \psi(\mathbf{x}) \rightarrow \varphi(\mathbf{x})$, then $I_{R}\left({ }_{R} I\right) \vDash D \varphi(\mathbf{x}) \rightarrow D \psi(\mathbf{x})$.

(i) follows from the definition of $D$ and (ii) is just [P, Proposition 8.20]. Proposition 2.2 says that $D$ is an anti-isomorphism between $\operatorname{Latt}\left({ }_{R} I\right)$ and $\operatorname{Latt}\left(I_{R}\right)$.

Example 2.3. If $\varphi(x) \Leftrightarrow r x=0$, then $D \varphi(x) \Leftrightarrow \exists v(x v)\left(\begin{array}{l}1 \\ r\end{array}\right)=0$ which is equivalent to $r \mid x$. Suppose that ${ }_{R} I \vDash r x=0 \rightarrow s x=0$. Then, since $R / R r \vDash$ $r(1+R r)=0$, it must be that $R / R r \vDash s(1+R r)=0$ i.e., that $s \in R r$ and that $s=\operatorname{tr}$ for some $t \in R$. But then it follows that $I_{R} \vDash s|x \rightarrow r| x$.

Since $D$ is inclusion reversing, ${ }_{R} I\left(I_{R}\right) \vDash D(\varphi(\mathbf{x}) \wedge \psi(\mathbf{x})) \leftrightarrow D \varphi(\mathbf{x})+D \psi(\mathbf{x})$, but one can easily check that when $\mathbf{x}$ and $\mathbf{y}$ are disjoint,

$$
{ }_{R} I\left(I_{R}\right) \vDash D(\varphi(\mathbf{x}) \wedge \psi(\mathbf{y})) \leftrightarrow D \varphi(\mathbf{x}) \wedge D \psi(\mathbf{y})
$$

so that some must be taken when computing the dual of $\varphi(\mathbf{x})$ as a formula in more than $l(\mathbf{x})$ variables. For if we take $l(x)=1(y)=1$ and $x \neq y$, then $D \varphi(x) \wedge y=0$ will be the dual of $\varphi(x)$ as a formula in two variables. 
B. The dual of $\rho(\mathbf{x}, \mathbf{y})$. We now turn out attention to ppfs of the form $\rho(\mathbf{x}, \mathbf{y})$. If we are working in ${ }_{R} L\left(L_{R}\right)$, we think of $\rho$ as acting on the left (right) so $\mathbf{x}$ is called the domain (codomain) variable and $\mathbf{y}$ the codomain (domain) variable. It is because of this perspective that the dual of $\rho(\mathbf{x}, \mathbf{y})$ is not simply the dual of $\rho(\mathbf{x} \wedge \mathbf{y})$ as given in Definition 2.1 , but is rather that dual with a twist.

Definition 2.4. The dual of

$$
\rho(\mathbf{x}, \mathbf{y}) \Leftrightarrow \exists \mathbf{w}\left(\begin{array}{lll}
H_{1} & H_{2} & H_{3}
\end{array}\right)\left(\begin{array}{c}
\mathbf{x} \\
\mathbf{y} \\
\mathbf{w}
\end{array}\right)=0
$$

is

$$
D \rho(\mathbf{x}, \mathbf{y}) \Leftrightarrow \exists \mathbf{v}(\mathbf{x y v})\left(\begin{array}{ccc}
I & 0 & 0 \\
0 & -I & 0 \\
H_{1} & H_{2} & H_{3}
\end{array}\right)=0 .
$$

If $\rho(\mathbf{x}, \mathbf{y}) \in L_{R}$ is given as

$$
\rho(\mathbf{x}, \mathbf{y}) \Leftrightarrow \exists \mathbf{v}(\mathbf{x y v})\left(\begin{array}{l}
H_{1} \\
H_{2} \\
H_{3}
\end{array}\right)=0,
$$

then

$$
D \rho(\mathbf{x}, \mathbf{y}) \Leftrightarrow \exists \mathbf{w}\left(\begin{array}{ccc}
I & 0 & H_{1} \\
0 & -I & H_{2} \\
0 & 0 & H_{3}
\end{array}\right)\left(\begin{array}{l}
\mathbf{x} \\
\mathbf{y} \\
\mathbf{w}
\end{array}\right)=0 .
$$

Example 2.5. If $\rho(x, y) \Leftrightarrow y=r x$, then $I_{R} \vDash D \rho(x, y) \leftrightarrow x=y r$ so that the dual of the action by $r \in R$ on left $R$-modules is the action by $r$ on the right.

We will often be interested in the dual of $\rho(\mathbf{x}, \mathbf{y})$ as a formula with just one sort of variable, i.e., we will want to take the dual of $\rho(\mathbf{x}, \mathbf{y})$ in the sense of Definition 2.1. In such cases we shall denote that dual by $D \rho\left(\mathbf{x}^{\wedge} \mathbf{y}\right)$. Note that in that case, ${ }_{R} I\left(I_{R}\right) \vDash D \rho(\mathbf{x}, \mathbf{y}) \leftrightarrow D \rho\left(\mathbf{x}^{\wedge}-\mathbf{y}\right)$. But $D \rho\left(\mathbf{x}^{\wedge}-\mathbf{y}\right)$ is ambiguous! It may be the dual $x$ in the sense of Definition 2.1) of the ppf $\rho\left(x^{\wedge}-y\right)$ or it may be $\varphi(\mathbf{x},-\mathbf{y})$ where $\varphi(\mathbf{x}, \mathbf{y}) \Leftrightarrow D \rho\left(\mathbf{x}^{\wedge} \mathbf{y}\right)$. It is easily verified that these two formulae are equivalent. These considerations and Proposition 2.2 yield

Proposition 2.6. (i) ${ }_{R} I\left(I_{R}\right) \vDash D^{2} \rho(\mathbf{x}, \mathbf{y}) \leftrightarrow \rho(\mathbf{x}, \mathbf{y})$ for each ppf $\rho(\mathbf{x}, \mathbf{y}) \in$ ${ }_{R} L\left(L_{R}\right)$.

(ii) If ${ }_{R} I\left(I_{R}\right) \vDash \rho(\mathbf{x}, \mathbf{y}) \rightarrow \sigma(\mathbf{x}, \mathbf{y})$, then $I_{R}\left({ }_{R} I\right) \vDash D \sigma(\mathbf{x}, \mathbf{y}) \rightarrow D \rho(\mathbf{x}, \mathbf{y})$.

C. The isomorphism. We begin by relating the duals of $\rho(\mathbf{x}, \mathbf{y})$ and $\varphi(\mathbf{y})$. As in the next proposition, we shall from time to time lapse into proving a result just for left $R$-modules when a dual argument suffices to prove that same result for the right side.

Proposition 2.7. For $\rho(\mathbf{x}, \mathbf{y})$ and $\varphi(\mathbf{y})$ in ${ }_{R} L\left(L_{R}\right)$,

$$
I_{R}\left({ }_{R} I\right) \vDash D(\exists \mathbf{y} \rho(\mathbf{x}, \mathbf{y}) \wedge \varphi(\mathbf{y})) \leftrightarrow \exists \mathbf{y}(D \rho(\mathbf{x}, \mathbf{y}) \wedge D \varphi(\mathbf{y})) .
$$

Proof. Assume that $\rho(\mathbf{x}, \mathbf{y})$ and $\varphi(\mathbf{y})$ are in ${ }_{R} L$. Let

$$
\rho(\mathbf{x}, \mathbf{y}) \Leftrightarrow \exists \mathbf{w}\left(\begin{array}{lll}
H_{1} & H_{2} & H_{3}
\end{array}\right)\left(\begin{array}{c}
\mathbf{x} \\
\mathbf{y} \\
\mathbf{w}
\end{array}\right)=0
$$


and let $\varphi(\mathbf{y}) \Leftrightarrow \exists \mathbf{v}\left(\begin{array}{ll}J_{1} & J_{2}\end{array}\right)\left(\begin{array}{l}\mathbf{y} \\ \mathbf{v}\end{array}\right)=0$. Then

$$
D \rho(\mathbf{x}, \mathbf{y}) \Leftrightarrow \exists \mathbf{w}_{0}\left(\mathbf{x y w}_{0}\right)\left(\begin{array}{ccc}
I & 0 & 0 \\
0 & -I & 0 \\
H_{1} & H_{2} & H_{3}
\end{array}\right)=0
$$

and

$$
D \varphi(\mathbf{y}) \Leftrightarrow \exists \mathbf{v}_{0}\left(\mathbf{y}_{0}\right)\left(\begin{array}{cc}
I & 0 \\
J_{1} & J_{2}
\end{array}\right)=0 .
$$

On the one hand we get

$$
\begin{aligned}
& I_{R} \vDash \exists \mathbf{y}(D \rho(\mathbf{x}, \mathbf{y}) \wedge D \varphi(\mathbf{y})) \\
& \quad \leftrightarrow \exists \mathbf{y}, \mathbf{v}_{0}, \mathbf{w}_{0}\left(\mathbf{x y v}_{0} \mathbf{w}_{0}\right)\left(\begin{array}{ccccc}
I & 0 & 0 & 0 & 0 \\
0 & -I & 0 & I & 0 \\
0 & 0 & 0 & J_{1} & J_{2} \\
H_{1} & H_{2} & H_{3} & 0 & 0
\end{array}\right)=0 \\
& \leftrightarrow \exists \mathbf{v}_{0}, \mathbf{w}_{0}\left(\mathbf{x v}_{0} \mathbf{w}_{0}\right)\left(\begin{array}{cccc}
I & 0 & 0 & 0 \\
0 & J_{1} & 0 & J_{2} \\
H_{1} & H_{2} & H_{3} & 0
\end{array}\right)=0,
\end{aligned}
$$

whereas

$$
{ }_{R} I \vDash(\exists \mathbf{y} \rho(\mathbf{x}, \mathbf{y}) \wedge \varphi(\mathbf{y})) \leftrightarrow \exists \mathbf{y}, \mathbf{w}, \mathbf{v}\left(\begin{array}{cccc}
H_{1} & H_{2} & H_{3} & 0 \\
0 & J_{1} & 0 & J_{2}
\end{array}\right)\left(\begin{array}{l}
\mathbf{x} \\
\mathbf{y} \\
\mathbf{w} \\
\mathbf{v}
\end{array}\right)=0
$$

so that

$$
I_{R} \vDash D(\exists \mathbf{y} \rho(\mathbf{x}, \mathbf{y}) \wedge \varphi(\mathbf{y})) \leftrightarrow \exists \mathbf{w}_{0}, \mathbf{v}_{0}\left(\mathbf{x w}_{0} \mathbf{v}_{0}\right)\left(\begin{array}{cccc}
I & 0 & 0 & 0 \\
H_{1} & H_{2} & H_{3} & 0 \\
0 & J_{1} & 0 & J_{2}
\end{array}\right)=0
$$

and the proposition follows.

Corollary 2.8. (i) $I_{R} \vDash D \rho(\mathbf{x}, 0) \leftrightarrow D(\exists \mathbf{y} \rho(\mathbf{x}, \mathbf{y}))$,

(ii) $I_{R} \vDash D(\rho(\mathbf{x}, 0)) \leftrightarrow \exists \mathbf{y} D \rho(\mathbf{x}, \mathbf{y})$,

(iii) $I_{R} \vDash D\left(\exists \mathbf{z} \rho_{1}(\mathbf{x}, \mathbf{z}) \wedge \rho_{2}(\mathbf{z}, \mathbf{y})\right) \leftrightarrow \exists \mathbf{z} D \rho_{1}(\mathbf{x}, \mathbf{z}) \wedge D \rho_{2}(\mathbf{z}, \mathbf{y})$.

Proof. (i) Let $\varphi(\mathbf{y}) \Leftrightarrow \mathbf{y}=\mathbf{y}$ in Proposition 2.7. Then $I_{R} \vDash D(\exists \mathbf{y} \rho(\mathbf{x}, \mathbf{y})) \leftrightarrow$ $\exists \mathbf{y}(D \rho(\mathbf{x}, \mathbf{y}) \wedge \mathbf{y}=0)$ and the result follows since $I_{R} \vDash \exists \mathbf{y}(D \rho(\mathbf{x}, \mathbf{y}) \wedge \mathbf{y}=0) \leftrightarrow$ $D \rho(\mathbf{x}, 0)$.

(ii) $I_{R} \vDash \exists \mathbf{y} D \rho(\mathbf{x}, \mathbf{y}) \leftrightarrow D^{2}\left(\exists \mathbf{y}(D \rho(\mathbf{x}, \mathbf{y})) \leftrightarrow D\left(D^{2} \rho(\mathbf{x}, 0)\right)\right.$, by (i). But $I_{R} \mid=D^{2} \rho(\mathbf{x}, 0) \leftrightarrow \rho(\mathbf{x}, 0)$.

(iii) Let $\sigma_{1}\left(\mathbf{x}^{\wedge} \mathbf{y}, \mathbf{z}\right) \Leftrightarrow \rho_{1}(\mathbf{x}, \mathbf{z}) \wedge \mathbf{y}=\mathbf{y}$ and let $\sigma_{2}(\mathbf{x} \wedge \mathbf{y}, \mathbf{z}) \Leftrightarrow \rho_{2}(\mathbf{z}, \mathbf{y}) \wedge \mathbf{x}=$ x. Then $I_{R} \vDash D \sigma_{1}\left(\mathbf{x}^{\wedge} \mathbf{y}, \mathbf{z}\right) \leftrightarrow D \rho_{1}(\mathbf{x}, \mathbf{z}) \wedge \mathbf{y}=0$ and $I_{R} \vDash D \sigma_{2}\left(\mathbf{x}^{\wedge} y, \mathbf{z}\right) \leftrightarrow$ $D \rho_{2}(\mathbf{z}, \mathbf{y}) \wedge \mathbf{x}=0$. Thus $I_{R} \vDash D\left(\exists \mathbf{z} \rho_{1}(\mathbf{x}, \mathbf{z}) \wedge \rho_{2}(\mathbf{z}, \mathbf{y})\right) \leftrightarrow D\left(\exists \mathbf{z} \sigma_{1}(\mathbf{x} \wedge \mathbf{y}, \mathbf{z}) \wedge\right.$ $\left.\sigma_{2}\left(\mathbf{x}^{\wedge} \mathbf{y}, \mathbf{z}\right)\right) \leftrightarrow D \sigma_{1}\left(\mathbf{x}^{\wedge} \mathbf{y}, \mathbf{z}\right)+\left.D \sigma_{2}\left(\mathbf{x}^{\wedge} \mathbf{y}, \mathbf{z}\right)\right|_{\mathbf{z}=0}$, by (i). But this is equivalent to the ppf $\exists \mathbf{x}_{1}, \mathbf{x}_{2}, \mathbf{y}_{1}, \mathbf{y}_{2}, \mathbf{z}_{1}, \mathbf{z}_{2} D \rho_{1}\left(\mathbf{x}_{1}, \mathbf{z}_{1}\right) \wedge \mathbf{y}_{1}=0 \wedge D \rho_{2}\left(\mathbf{z}_{2}, \mathbf{y}_{2}\right) \wedge \mathbf{x}_{2}=$ $0 \wedge \mathbf{x}=\mathbf{x}_{1}+\mathbf{x}_{2} \wedge \mathbf{y}=\mathbf{y}_{1}+\mathbf{y}_{2} \wedge \mathbf{z}=\mathbf{z}_{1}+\mathbf{z}_{2}=0$ which reduces to the ppf $\exists \mathbf{z} D \rho_{1}(\mathbf{x}, \mathbf{z}) \wedge D \rho_{2}(\mathbf{z}, \mathbf{y})$.

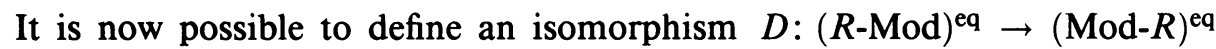
by letting $D(\varphi / \psi)=D \psi / D \varphi$ and if $\rho \in \operatorname{Mor}(\varphi / \psi, \sigma / \tau)$ is represented by 
$\rho(\mathbf{x}, \mathbf{y})$, then $D \rho \in \operatorname{Mor}(D \psi / D \varphi, D \tau / D \sigma)$ is represented by $D \rho(\mathbf{x}, \mathbf{y})$. We just need to show that this makes sense. If we apply $D$ to the four conditions which assure that $\rho(\mathbf{x}, \mathbf{y})$ represents a natural transformation we get, by use of Proposition 2.2 and Corollary 2.8, the following implications:

(i) ${ }_{R} I \vDash \varphi(\mathbf{x}) \rightarrow \exists \mathbf{y} \rho(\mathbf{x}, \mathbf{y})$ implies (i') $I_{R} \vDash D \rho(\mathbf{x}, 0) \rightarrow D \varphi(\mathbf{x})$,

(ii) ${ }_{R} I \vDash \psi(\mathbf{x}) \rightarrow \rho(\mathbf{x}, 0)$ implies (ii') $I_{R} \vDash \exists \mathbf{y} D \rho(\mathbf{x}, \mathbf{y}) \rightarrow D \psi(\mathbf{x})$,

(iii) ${ }_{R} I \vDash \exists \mathbf{x} \rho(\mathbf{x}, \mathbf{y}) \rightarrow \sigma(\mathbf{y})$ implies (iii') $I_{R} \vDash D \sigma(\mathbf{y}) \rightarrow D \rho(\mathbf{x}, 0)$,

(iv) ${ }_{R} I \vDash \rho(0, \mathbf{y}) \rightarrow \tau(\mathbf{y})$ implies $\left(\right.$ iv $\left.^{\prime}\right) I_{R} \vDash D \tau(\mathbf{y}) \rightarrow \exists \mathbf{x} D \rho(\mathbf{x}, \mathbf{y})$.

But $\left(\mathbf{i}^{\prime}\right)-\left(\mathbf{i v}^{\prime}\right)$ are exactly the properties (permuted) that $D \rho(\mathbf{x}, \mathbf{y})$ must have in order to represent a natural transformation $D \rho \in \operatorname{Mor}(D \psi / D \varphi, D \tau / D \sigma)$.

Theorem 2.9. $D:(R-\mathrm{Mod})^{\mathrm{eq}} \cong(\operatorname{Mod}-R)^{\mathrm{eq}}$.

Proof. By Propositions 2.2 and $2.6, D^{2}=$ identity so we just need to show that $D$ respects composition of morphisms. In other words, we must prove that $D\left(\rho_{2} \rho_{1}\right)=D \rho_{2} D \rho_{1}, \rho_{2} \rho_{1}$ may be represented by the formula $\left(\rho_{2} \rho_{1}\right)(\mathbf{x}, \mathbf{y}) \Leftrightarrow$ $\exists \mathbf{z} \rho_{1}(\mathbf{x}, \mathbf{z}) \wedge \rho_{2}(\mathbf{z}, \mathbf{y})$. Applying $D$ and Corollary 2.8(iii), we get

$$
\begin{aligned}
I_{R} \vDash D\left(\rho_{2} \rho_{1}\right)(\mathbf{x}, \mathbf{y}) & \leftrightarrow D\left(\exists \mathbf{z} \rho_{1}(\mathbf{x}, \mathbf{z}) \wedge \rho_{2}(\mathbf{z}, \mathbf{y})\right) \\
& \leftrightarrow\left(\exists \mathbf{z} D \rho_{1}(\mathbf{x}, \mathbf{z}) \wedge D \rho_{2}(\mathbf{z}, \mathbf{y})\right),
\end{aligned}
$$

a ppf which represents $D \rho_{2} D \rho_{1}$.

\section{TENSOR, TENSORS!}

To show that the definition of duality is a natural one, we shall explore how duality behaves under tensoring. Example 2.3 has already hinted at what happens to $\varphi\left({ }_{R} M\right)$ and $D \varphi\left(N_{R}\right)$ in $N \otimes_{R} M$. One by-product of our considerations will be that it is possible to define tensoring sortwise. $\sum\left\{x_{i} \otimes y_{i} \mid 0 \leq i<l(\mathbf{x})=\right.$ $l(\mathbf{y})\}$ will be denoted by $\mathbf{x} \otimes \mathbf{y}$. Note: all tensors in this paper are over $R$.

Lemma 3.1 [S, I.8.8] Let $\left\{y_{i} \mid i \in I\right\}$ be a family of generators of ${ }_{R} M$ and let $\left\{x_{i} \mid i \in I\right\} \in N_{R}$ with almost all $x_{i}=0$. Then $\sum\left\{x_{i} \otimes y_{i} \mid i \in I\right\}=0$ in $N \otimes_{R} M$ iff there exists a finite family $\left\{u_{j} \mid j \in J\right\}$ of elements of $N_{R}$ and row-finite matrix $H$ with $|J|$ rows and entries in $R$ so that

(i) $H \mathbf{y}=0$

(ii) $\mathbf{x}=\mathbf{u} H$.

The model-theoretic formulation of Lemma 3.1 is nicer.

Proposition 3.2. If $\mathbf{a} \in N_{R}$ and $\mathbf{c} \in{ }_{R} M$ are tuples of the same length, then $\mathbf{a} \otimes \mathbf{c}=0$ in $N \otimes_{R} M$ iff there is a ppf $\varphi(\mathbf{x}) \in{ }_{R} L$ such that ${ }_{R} M \vDash \varphi(\mathbf{c})$ and $N_{R} \vDash D \varphi(\mathbf{a})$.

Proof. Suppose that $\mathbf{a} \otimes \mathbf{c}=0$. By Lemma 3.1, there is an $\mathbf{e} \in{ }_{R} M$ (a finite subfamily of an extension of $\mathrm{c}$ to a generating family for ${ }_{R} M$ and a finite matrix $H=\left(\begin{array}{lll}H_{1} & H_{2}\end{array}\right)$ such that $\left(H_{1} H_{2}\right)\left(\begin{array}{l}\mathbf{c} \\ \mathbf{e}\end{array}\right)=0$ and there exists $\mathbf{u} \in N_{R}$ such that $(\mathbf{a}, 0)=\mathbf{u}\left(H_{1} H_{2}\right)$. So if $\varphi(\mathbf{x}) \Leftrightarrow \exists \mathbf{v}\left(H_{1} H_{2}\right)\left(\begin{array}{l}\mathbf{x} \\ \mathbf{v}\end{array}\right)=0$ then ${ }_{R} M \vDash \varphi(\mathbf{c})$ and since $N_{R} \vDash \mathbf{a}=\mathbf{u} H_{1} \wedge \mathbf{u} H_{2}=0$ we have that $N_{R} \vDash(\mathbf{a}-\mathbf{u})\left(\begin{array}{cc}I & 0 \\ H_{1} & H_{2}\end{array}\right)=0$ and hence $N_{R} \vDash D \varphi(\mathbf{a})$. 
If $R_{R} M \vDash \varphi(\mathbf{c})$ and $N_{R} \vDash D \varphi(\mathbf{a})$ with $\varphi(\mathbf{x}) \Leftrightarrow \exists \mathbf{v}\left(\begin{array}{ll}H_{1} & H_{2}\end{array}\right)\left(\begin{array}{l}\mathbf{x} \\ \mathbf{v}\end{array}\right)=0$, then

$$
N_{R} \vDash \exists \mathbf{u}(\mathbf{a u})\left(\begin{array}{cc}
I & 0 \\
H_{1} & H_{2}
\end{array}\right)=0 .
$$

Thus $\mathbf{a} \otimes \mathbf{c}=-\mathbf{u} H_{1} \otimes \mathbf{c}=-\mathbf{u} \otimes H_{1} \mathbf{c}=-\mathbf{u} \otimes-H_{2} \mathbf{v}=\mathbf{u} H_{2} \otimes \mathbf{v}=0 \otimes \mathbf{v}=0$.

Corollary 3.3. $\mathbf{a} \otimes \mathbf{d}=\mathbf{c} \otimes \mathbf{b}$ in $N \otimes_{R} M$ iff there is a ppf $\rho(\mathbf{x}, \mathbf{y})$ such that ${ }_{R} M \vDash \rho(\mathbf{d}, \mathbf{b})$ and $N_{R} \vDash D \rho(\mathbf{a}, \mathbf{c})$. We shall write this as $\mathbf{c} D \rho \otimes \mathbf{d}=\mathbf{c} \otimes \rho \mathbf{d}$.

The last statement of Corollary 3.3 is telling, for it says that one can "pass over" the tensor not only with the action of $R$, but with any pp-defined action. Now pp-action is in general only partially defined and even then it is all too often many-valued. This is why we choose as our objects of study the pairs $\varphi / \psi$, for it is on an appropriate pp-pair that a pp-action becomes a (total, well-defined) function.

Corresponding to each $\varphi / \psi \in \mathrm{Ob}(R-\mathrm{Mod})^{\text {eq }}$, we can define a subfunctor of - $\otimes$-, which takes $N_{R} \times{ }_{R} M \in \mathrm{Ob}\left(\operatorname{Mod}-R \times R\right.$-Mod) into $D \psi(N) \otimes_{R} \varphi(M)$, the abelian subgroup of $N \otimes_{R} M$ generated by the set $\left\{\left.\mathbf{a} \otimes \mathbf{c}\right|_{R} M \vDash \varphi(\mathbf{c})\right.$ and $\left.N_{R} \vDash D \psi(\mathbf{a})\right\}$. That this is indeed a functor is easily verified and we denote it by $D \psi \otimes \varphi$. This illustrates that it is in $M^{\text {eq }}$ that tensoring really takes place. If, moreover, we set $R(\varphi / \psi)=\operatorname{Mor}(\varphi / \psi, \varphi / \psi)$, then a special case of Theorem 2.9 is

Proposition 3.4. $R(\varphi / \psi) \cong R(D \psi / D \varphi)$ as rings.

Proof. We just need to check that the duality $D$ respects addition. If $\rho_{1}, \rho_{2} \in$ $R(\varphi / \psi)$, then $\rho_{1}+\rho_{2}$ is represented by

$$
\left(\rho_{1}+\rho_{2}\right)(\mathbf{x}, \mathbf{y}) \Leftrightarrow \exists \mathbf{z} \rho_{1}(\mathbf{x}, \mathbf{z}) \wedge \rho_{2}(\mathbf{x}, \mathbf{y}-\mathbf{z}) .
$$

It is straightforward to check that this does indeed conform to the conditions for being an element of $R(\varphi / \psi)$. We aim to show that $I_{R} \vDash D\left(\rho_{1}+\rho_{2}\right)(\mathbf{x}, \mathbf{y}) \leftrightarrow$ $\left(D \rho_{1}+D \rho_{2}\right)(\mathbf{x}, \mathbf{y})$. To prove this, let $\sigma_{1}\left(\mathbf{x}^{\wedge} \mathbf{y}, \mathbf{z}\right) \Leftrightarrow \rho_{1}(\mathbf{x}, \mathbf{z}) \wedge \mathbf{y}=\mathbf{y}$ and let $\sigma_{2}\left(\mathbf{x}^{\wedge} \mathbf{y}, \mathbf{z}\right) \Leftrightarrow \rho_{2}(\mathbf{x}, \mathbf{y}-\mathbf{z})$. Then

$$
I_{R} \vDash D \sigma_{1}\left(\mathbf{x}^{\curlyvee} \mathbf{y}, \mathbf{z}\right) \leftrightarrow D \rho_{1}(\mathbf{x}, \mathbf{z}) \wedge \mathbf{y}=0
$$

and

$$
I_{R} \vDash D \sigma_{2}\left(\mathbf{x}^{\wedge} \mathbf{y}, \mathbf{z}\right) \leftrightarrow D \rho_{2}(\mathbf{x},-\mathbf{y}) \wedge \mathbf{z}=\mathbf{y}
$$

Thus

$$
\begin{aligned}
I_{R} \vDash D\left(\rho_{1}+\rho_{2}\right)\left(\mathbf{x}^{\curlywedge} \mathbf{y}\right) & \leftrightarrow D\left(\exists \mathbf{z} \sigma_{1}\left(\mathbf{x}^{\wedge} \mathbf{y}, \mathbf{z}\right) \wedge \sigma_{2}\left(\mathbf{x}^{\wedge} \mathbf{y}, \mathbf{z}\right)\right) \\
& \leftrightarrow D \sigma_{1}\left(\mathbf{x}^{\wedge} \mathbf{y}, \mathbf{z}\right)+\left.D \sigma_{2}\left(\mathbf{x}^{\wedge} \mathbf{y}, \mathbf{z}\right)\right|_{\mathbf{z}=0}
\end{aligned}
$$

by Corollary 2.8(i). But this is equivalent to the ppf $\exists \mathbf{x}_{1}, \mathbf{x}_{2}, \mathbf{y}_{1}, \mathbf{y}_{2}, \mathbf{z}_{1}, \mathbf{z}_{2}$ $D \rho_{1}\left(\mathbf{x}_{1}, \mathbf{z}_{1}\right) \wedge \mathbf{y}_{1}=0 \wedge D \rho_{2}\left(\mathbf{x}_{2},-\mathbf{y}_{2}\right) \wedge \mathbf{z}_{2}=\mathbf{y}_{2} \wedge \mathbf{x}=\mathbf{x}_{1}+\mathbf{x}_{2} \wedge \mathbf{y}=\mathbf{y}_{1}+\mathbf{y}_{2} \wedge \mathbf{z}=$ $\mathbf{z}_{1}+\mathbf{z}_{2}=0$ which reduces to the ppf $\exists \mathbf{z} D \rho_{1}(\mathbf{x}-\mathbf{z},-\mathbf{y}) \wedge D \rho_{2}(\mathbf{z},-\mathbf{y})$. It follows that $I_{R} \vDash D\left(\rho_{1}+\rho_{2}\right)(\mathbf{x}, \mathbf{y}) \leftrightarrow D\left(\rho_{1}+\rho_{2}\right)\left(\mathbf{x}^{\wedge}-\mathbf{y}\right) \leftrightarrow \exists \mathbf{z} D \rho_{1}(\mathbf{x}-\mathbf{z}, \mathbf{y}) \wedge D \rho_{2}(\mathbf{z}, \mathbf{y}) \leftrightarrow$ $\left(D \rho_{1}+D \rho_{2}\right)(\mathbf{x}, \mathbf{y})$.

If we identify these two rings, simply calling it $R(\varphi / \psi)$, then $\varphi / \psi(M)$ is naturally endowed with the structure of a left $R(\varphi / \psi)$-module and $D \psi / D \varphi(N)$ 
can be thought of as a right $R(\varphi / \psi)$-module. All that said, we can illustrate the above scenario with the diagram

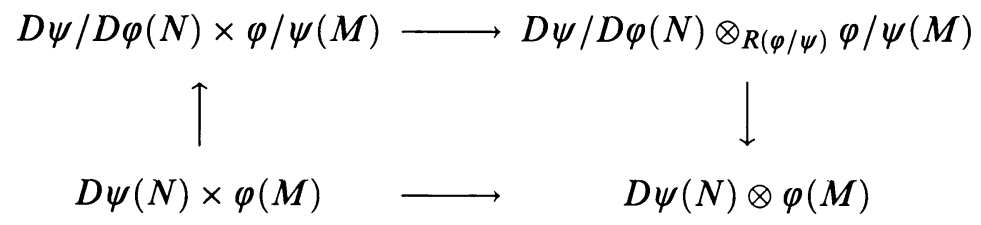

which is commutative and whose vertical arrows represent surjections.

In order to make some basic observations about the function which sends the functor $\varphi / \psi$ to the functor $D \psi \otimes \varphi$ we need to make the following definition.

Definition 3.5. $\varphi / \psi \leq \sigma / \tau$ if there is a $\rho \in \operatorname{Mor}(\varphi / \psi, \sigma / \tau)$ such that $\rho$ : $\varphi(M) / \psi(M) \rightarrow \sigma(M) / \tau(M)$ is an imbedding for each $M \in R$-Mod.

If $\rho(\mathbf{x}, \mathbf{y})$ represents some $\rho \in \operatorname{Mor}(\varphi / \psi, \sigma / \tau)$ which witnesses $\varphi / \psi \leq$ $\sigma / \tau$, it is easily seen that in addition to the usual properties of $\rho(\mathbf{x}, \mathbf{y})$, we have that ${ }_{R} I \vDash \psi(\mathbf{x}) \leftrightarrow \rho(\mathbf{x}, 0)$.

Proposition 3.6. (i) $\exists \mathbf{y} D \rho(\mathbf{x}, \mathbf{y})(N) \otimes \exists \mathbf{y} \rho(\mathbf{x}, \mathbf{y})(M) \subseteq \exists \mathbf{x} D \rho(\mathbf{x}, \mathbf{y})(N) \otimes \exists \mathbf{x}$ $\rho(\mathbf{x}, \mathbf{y})(M)$.

(ii) If $\varphi / \psi \leq \sigma / \tau$, then $D \psi(N)_{R} \otimes \varphi(M) \subseteq D \tau(N) \otimes_{R} \sigma(M)$.

Proof. (i) Let $\mathbf{a} \in M$ such that $M \vDash \exists \mathbf{y} \rho(\mathbf{a}, \mathbf{y})$ and let $\mathbf{b} \in M$ witness this and take $\mathbf{c} \in N$ for which $N \vDash \exists \mathbf{y} D \rho(\mathbf{c}, \mathbf{y})$ and let $\mathbf{e} \in N$ be a witness. It is enough to prove that $\mathbf{c} \otimes \mathbf{a}$ is in $\exists \mathbf{x} D \rho(\mathbf{x}, \mathbf{y})(N) \otimes \exists \mathbf{x} \rho(\mathbf{x}, \mathbf{y})(M)$. But Corollary 3.3 implies that $\mathbf{c} \otimes \mathbf{a}=\mathbf{e} D \rho \otimes \mathbf{a}=\mathbf{e} \otimes \rho \mathbf{a}=\mathbf{e} \otimes \mathbf{b}$ where $M \vDash \exists \mathbf{x} \rho(\mathbf{x}, \mathbf{b})$ and $N \vDash \exists \mathbf{x} D \rho(\mathbf{x}, \mathbf{e})$ so the result follows.

(ii) Let $\rho(\mathbf{x}, \mathbf{y})$ represent some $\rho \in \operatorname{Mor}(\varphi / \psi, \sigma / \tau)$ which witnesses $\varphi / \psi \leq$ $\sigma / \tau$. We have noted above that, in this case, ${ }_{R} I \vDash \psi(\mathbf{x}) \leftrightarrow \rho(\mathbf{x}, 0)$, so from Corollary 2.8 we get that $I_{R} \vDash D \psi(\mathbf{x}) \leftrightarrow \exists \mathbf{y} D \rho(\mathbf{x}, \mathbf{y})$. By (i) and the properties of $\rho(\mathbf{x}, \mathbf{y})$ we see that $D \psi(N)_{R} \otimes \varphi(M) \subseteq \exists \mathbf{x} D \rho(\mathbf{x}, \mathbf{y})(N) \otimes \sigma(M)$. Since ${ }_{R} I \vDash \tau(\mathbf{y}) \leftrightarrow \rho(0, \mathbf{y})$, another application of Corollary 2.8 gives $I_{R} \vDash D \tau(\mathbf{y}) \leftrightarrow$ $\exists \mathbf{x} D \rho(\mathbf{x}, \mathbf{y})$ and the proposition is proved.

Now that we have defined tensoring sortwise, we are forced to consider sortwise homing. Lest the notation become overly oppressive, we shall assume for the rest of this section that $\varphi / \psi$ is a pp-pair of formulae in one variable. Of course, what follows may be applied to arbitrary pp-pairs. Let $\overline{\mathbf{m}}$ be a tuple of elements from $\varphi / \psi(M)$, then each $m \in \mathbf{m}$ is in $\varphi(M)$ and $\bar{m} \in \overline{\mathbf{m}}$ is its image in $\varphi / \psi(M)$ under the natural projection.

Definition 3.7. If (i) $\overline{\mathbf{m}} \in \varphi / \psi(M)$ has $l(\overline{\mathbf{m}})=n$, then $\operatorname{tp}^{+}(\overline{\mathbf{m}})=\{\sigma(\mathbf{x}) \in$ $\operatorname{tp}^{+}(\mathbf{m}) \mid \vDash \psi^{n}(\mathbf{x}) \rightarrow \sigma(\mathbf{x})$ and $\left.\vDash \sigma(\mathbf{x}) \rightarrow \varphi^{n}(\mathbf{x})\right\}$.

(ii) For $R$-modules $M$ and $N, f: \varphi / \psi(M) \rightarrow \varphi / \psi(N)$ is a member of $\operatorname{Hom}_{R}(\varphi / \psi(M), \varphi / \psi(N))$ iff $\operatorname{tp}^{+}(\overline{\mathbf{m}} f) \supseteq \operatorname{tp}^{+}(\overline{\mathbf{m}})$ for each $\overline{\mathbf{m}} \in \varphi / \psi(M)$.

We shall refer to $f \in \operatorname{Hom}_{R}(\varphi / \psi(M), \varphi / \psi(N))$ as a sort homomorphism and if $M=N$, then we call $f$ a sort endomorphism of $M$. The only fact about sort endomorphisms which we shall need is the following

Proposition 3.8. If $M$ is pure-injective, then each sort endomorphism of $M$ is induced by an endomorphism of $M$. 
Proof. Let $f \in \operatorname{Hom}_{R}(\varphi / \psi(M), \varphi / \psi(M))$ and let $\mathbf{c} \in C=\varphi(M), l(\mathbf{c})=n$. For each $c \in$ c choose $c_{f} \in \varphi(M)$ such that $(c+\psi(M)) f=c_{f}+\psi(M)$ and let $\mathbf{c}_{f}=\left(c_{f} \mid c \in \mathbf{c}\right)$. Consider $\sigma(\mathbf{x}) \in \operatorname{tp}^{+}(\mathbf{c})$ such that $\vDash \sigma(\mathbf{x}) \rightarrow \varphi^{n}(\mathbf{x})$. Then $\sigma(\mathbf{x})+\psi^{n}(\mathbf{x}) \in \operatorname{tp}^{+}(\overline{\mathbf{c}})$ and so, by hypothesis, $\sigma(\mathbf{x})+\psi^{n}(\mathbf{x}) \in \operatorname{tp}^{+}(\overline{\mathbf{c}} f)$ and hence $\sigma(\mathbf{x})+\psi^{n}(\mathbf{x}) \in \operatorname{tp}^{+}\left(\mathbf{c}_{f}\right)$. This means that $\operatorname{tp}^{+}(\mathbf{c}) \cup\left\{\psi\left(\mathbf{x}-\mathbf{c}_{f}\right)\right\}$ is finitely satisfiable in $M$ and hence that $\operatorname{tp}^{+}(C) \cup\left\{\psi\left(x_{c}-c_{f}\right) \mid c \in C\right\}$ is finitely satisfiable in $M$. As $M$ is pure-injective, Proposition 0.4 says that $\operatorname{tp}^{+}(C) \cup\left\{\psi\left(x-c_{f}\right) \mid c \in C\right\}$ is realized in $M$ by, say, $\{c \tilde{f} \mid c \in C\}$. But then the function $\tilde{f}$ defined on $\varphi(M)$ which takes $c$ to $c \tilde{f}$ is a partial homomorphism from $M$ to itself and hence, by [Z, Corollary 3.3], lifts to an endomorphism $\tilde{f}$ of $M$. For $c \in \varphi(M)$ we have that $M \vDash \psi\left(c \tilde{f}-c_{f}\right)$ and so $\tilde{f}$ induces on $\varphi / \psi(M)$ the sort endomorphism which behaves as $(c+\psi(M)) \tilde{f}=c \tilde{f}+\psi(M)=$ $c_{f}+\psi(M)=(c+\psi(M)) f$ and so must be equal to $f$.

\section{The ZiEgLER SPECTRUM}

In this section, we use Theorem 2.9 to relate the left and right Ziegler spectra. Some conventions need to be set. If $\Phi(\mathbf{x})$ is a filter of ppfs in the variable $\mathbf{x}$, then we shall often confuse $\Phi(\mathbf{x})$ with the infinitary formula $\Lambda\{\varphi(\mathbf{x}) \mid \varphi(\mathbf{x}) \in$ $\Phi(\mathbf{x})\}$. Dually, an ideal $\Psi$ of ppfs i.e., a downward closed set which is also closed under + , will be confused with $\sum\{\psi(\mathbf{x}) \mid \psi(\mathbf{x}) \in \Psi(\mathbf{x})\}$. For $\Phi(\mathbf{x})$, a filter as above, $D \Phi(\mathbf{x})$ will denote the ideal $\{D \varphi(\mathbf{x}) \mid \varphi(\mathbf{x}) \in \Phi(\mathbf{x})\}$ and dually. We need a

Lemma 4.1. If $\Phi \subseteq{ }_{R} L\left(L_{R}\right)$ is a filter of ppfs and $\Psi \subseteq{ }_{R} L\left(L_{R}\right)$ is an ideal, maximal with respect to the property that $\varphi \mid \varphi \in \Phi \cup\{\neg \psi \mid \psi \in \Psi\}=\varnothing$ is consistent $\left(\right.$ in $\left.{ }_{R} T\right)$, then there is an indecomposable pp-type $p(\mathbf{x}) \subseteq L_{R}\left({ }_{R} L\right)$ such that $D \Psi=p^{+}(\mathbf{x})$. In particular, $p^{-}(\mathbf{x}) \supseteq D \Phi$.

Proof. Given the hypotheses, $D \Psi$ is a filter of ppfs maximal with respect to the property $\{D \psi \mid D \psi \in D \Psi\} \cap\{\neg D \varphi \mid D \varphi \in D \Phi\}=\varnothing$ (in $T_{R}$ ). The lemma then follows immediately from [P, Theorem 4.33].

Definition 4.2. Let $O \subseteq{ }_{R} I\left(I_{R}\right)$ be open. Then the reflection of $O, O^{\prime}=$ $\bigcup\{(D \psi / D \psi) \mid(\varphi / \psi) \subseteq O\} \subseteq I_{R}\left({ }_{R} I\right)$.

It is clear from the definition that ' respects $\subseteq$.

Lemma 4.3. $(\varphi / \psi) \subseteq \bigcup\left\{\left(\varphi_{i} / \psi_{i}\right) \mid 1 \leq i \leq n\right\}$ implies

$$
(D \psi / D \varphi) \subseteq \bigcup\left\{\left(D \psi_{i} / D \varphi_{i}\right) \mid 1 \leq i \leq n\right\} .
$$

Proof. Suppose to the contrary. Let $U_{R} \in(D \psi / D \varphi) \backslash \bigcup\left\{\left(D \psi_{i} / D \varphi_{i}\right) \mid 1 \leq i \leq n\right\}$ and take $\mathbf{a} \in D \psi(U) \backslash D \varphi(U)$. Let $D \Psi(\mathbf{x})=\operatorname{tp}^{+}(\mathbf{a})$ and let $D \Phi(\mathbf{x})$ be an ideal maximal with respect to the properties $D \varphi(\mathbf{x}) \in D \Phi$ and $D \Phi \cap D \Psi=\varnothing$. By Lemma 4.1, $\Phi(\mathbf{x})=\operatorname{tp}^{+}(\mathbf{c})$ for some $\mathbf{c}$ with $H(\mathbf{c})=V_{R}$ indecomposable. As $\Psi \subseteq \operatorname{tp}^{-}(\mathrm{c})$, we get that $\mathrm{c} \in \varphi(V) \backslash \psi(V)$ and so $V \in(\varphi / \psi)$. By hypothesis, there is an $i, 1 \leq i \leq n$, such that $V \in\left(\varphi_{i} / \psi_{i}\right)$. As in the proof of $[\mathrm{Z}$, Theorem 4.9], there is a ppf $\sigma(\mathbf{x}, \mathbf{y})$ such that

$$
\frac{\exists \mathbf{y} \sigma(\mathbf{x}, \mathbf{y}) \wedge \varphi_{i}(\mathbf{y})}{\exists \mathbf{y} \sigma(\mathbf{x}, \mathbf{y}) \wedge \psi_{i}(\mathbf{y})} \in \mathrm{pp}-\operatorname{tp}(\mathbf{c})
$$


and it follows that $D\left(\exists \mathbf{y} \sigma(\mathbf{x}, \mathbf{y}) \wedge \varphi_{i}(\mathbf{y})\right) \in D \Phi$ and hence by Proposition 2.7, $\exists \mathbf{y} D \sigma(\mathbf{x}, \mathbf{y}) \wedge D \varphi_{i}(\mathbf{y}) \in D \Phi$. Since $D \psi_{i}(U) / D \varphi_{i}(U)=0, U \vDash \exists \mathbf{y} D \sigma(\mathbf{x}, \mathbf{y}) \wedge$ $D \psi_{i}(\mathbf{y}) \rightarrow \exists \mathbf{y} D \sigma(\mathbf{x}, \mathbf{y}) \wedge D \varphi_{i}(\mathbf{y})$. This means that the ideal generated by $D \Phi$ and $\left\{\exists \mathbf{y} D \sigma(\mathbf{x}, \mathbf{y}) \wedge D \psi_{i}(\mathbf{y})\right\}$ is contained in $\operatorname{tp}^{-}(\mathbf{a})$. By maximality of $D \Phi$, $\exists \mathbf{y} D \sigma(\mathbf{x}, \mathbf{y}) \wedge D \psi_{i}(\mathbf{y}) \in D \Phi$, so applying $D, D\left(\exists \mathbf{y} D \sigma(\mathbf{x}, \mathbf{y}) \wedge D \psi_{i}(\mathbf{y})\right) \in \Phi$. Another application of Proposition 2.7 shows that $\exists \mathbf{y} \sigma(\mathbf{x}, \mathbf{y}) \wedge \psi_{i}(\mathbf{y}) \in \Phi=$ $\operatorname{tp}^{+}(\mathbf{c})$, a contradiction.

Lemma 4.3 is enough to prove that the topologies on ${ }_{R} I$ and $I_{R}$ are the same in the following sense.

Proposition 4.4. (i) $(\varphi / \psi)^{\prime}=(D \psi / D \varphi)$,

(ii) $O^{\prime \prime}=O$,

(iii) $\left(\bigcup\left\{O_{i} \mid i \in I\right\}\right)^{\prime}=\bigcup\left\{O_{i}^{\prime} \mid i \in I\right\}$,

(iv) $\left(\bigcap\left\{O_{i} \mid 1 \leq i \leq n\right\}\right)^{\prime}=\bigcap\left\{O_{i}^{\prime} \mid 1 \leq i \leq n\right\}$.

Proof. (i) $(D \psi / D \varphi) \subseteq(\varphi / \psi)^{\prime}$ follows from the definition of ' , so it is enough to prove that $(D \psi / D \varphi) \supseteq(\varphi / \psi)^{\prime}$. Suppose that $(\sigma / \tau) \subseteq(\varphi / \psi)$. Then Lemma 4.2 implies $(D \tau / D \sigma) \subseteq(D \psi / D \varphi)$ so that $(\varphi / \psi)^{\prime}=\bigcup\{(D \tau / D \sigma) \mid(\sigma / \tau) \subseteq$ $(\varphi / \psi)\} \subseteq(D \psi / D \varphi)$.

(ii) $O \subseteq O^{\prime \prime}$ is clear. To prove $O^{\prime \prime} \subseteq O$, let $(\varphi / \psi) \subseteq O^{\prime \prime}=\bigcup\{(\sigma / \tau) \mid(D \tau / D \sigma)$ $\left.\subseteq O^{\prime}\right\}$. By compactness of $(\varphi / \psi)$, there are $\left(D \tau_{i} / D \sigma_{i}\right) \subseteq O^{\prime}$ for $1 \leq i \leq n$ such that $(\varphi / \psi) \subseteq \bigcup\left\{\left(\sigma_{i} / \tau_{i}\right) \mid 1 \leq i \leq n\right\}$. Lemma 4.2 yields $(D \psi / D \varphi) \subseteq$ $\bigcup\left\{\left(D \tau_{i} / D \sigma_{i}\right) \mid 1 \leq i \leq n\right\} \subseteq O^{\prime}$. Repeating this whole argument gives that $(\varphi / \psi) \subseteq O$. By (ii), the lattices of open sets are isomorphic, whence (iii) and (iv).

Thus the topologies on ${ }_{R} I$ and $I_{R}$ are indeed the same. We do not mean to say that ${ }_{R} I$ and $I_{R}$ are homeomorphic, although this point will be addressed shortly. We shall refer to any action of ${ }^{\prime}$ as reflection. So, for example, for $I \subseteq{ }_{R} I\left(I_{R}\right)$ a closed set, its reflection $I^{\prime}$ is defined via complements, i.e., $I^{\prime}=I_{R} \backslash\left({ }_{R} I \backslash I\right)^{\prime}\left({ }_{R} I \backslash\left(I_{R} \backslash I\right)^{\prime}\right)$. The relationship between $I$ and $I^{\prime}$ is as expected.

Proposition 4.5. (i) $I \vDash|\varphi / \psi|>1$ iff $I^{\prime} \vDash|D \psi / D \varphi|>1$,

(ii) $\operatorname{Latt}\left(I^{\prime}\right) \cong \operatorname{Latt}(I)^{\text {op }}$

Proof. (i) By use of Corollary 4.4 and the definition of $I^{\prime}, I \vDash|\varphi / \psi|>1$ iff $I \cap(\varphi / \psi) \neq \varnothing$ iff $I^{\prime} \cap(\varphi / \psi)^{\prime} \neq \varnothing$ iff $I \cap(D \psi / D \varphi) \neq \varnothing$ iff $I^{\prime} \vDash|D \psi / D \varphi|>1$.

(ii) The function which takes $\varphi \in \operatorname{Latt}(I)$ to $D \varphi \in \operatorname{Latt}\left(I^{\prime}\right)$ is inclusion reversing by (i) and it has an inverse, $D$.

There are a number of ordinal (or $\infty$ )-valued measures of complexity which may be placed on a lattice and which attain the same value for a given lattice and its opposite. We have already encountered $m$-dimension in $\S 0$ and breadth and width [P, Chapter 8] are other examples of such measures. Proposition 4.4 then implies $m-\operatorname{dim}(I)=m-\operatorname{dim}\left(I^{\prime}\right)\left(\right.$ and $\operatorname{br}(I)=\operatorname{br}\left(I^{\prime}\right)$ and $\left.w(I)=w\left(I^{\prime}\right)\right)$.

Although we shall not give a complete treatment of localization until $\S 6$, the following lemma will be our first local statement. It will serve to prove local versions of things proved by Lemma 4.1.

Lemma 4.6 (local version of Lemma 4.1). Let $T$ be a complete theory of left (right) $R$-modules and $\Phi \subseteq{ }_{R} L\left(L_{R}\right)$ a filter of ppfs and $\Psi \subseteq{ }_{R} L\left(L_{R}\right)$ and ideal, 
maximal with respect to the property that $\Phi \cup\{\neg \psi \mid \psi \in \Psi\}$ is T-consistent. Then there is an indecomposable pp-type $p(\mathbf{x}) \subseteq L_{R}\left({ }_{R} L\right)$ such that $D \Psi=p^{+}(\mathbf{x})$ and $H(p) \in I(T)^{\prime}$.

Proof. Let $T^{\prime}=T\left(I(T)^{\prime}\right)$. Then $D \Psi$ is a filter of ppfs, maximal with respect to the property that $D \Psi \cup\{\neg D \varphi \mid \varphi \in \Phi\}$ is $T^{\prime}$-consistent. If we relativize [P, Theorem 4.33] to $T^{\prime}$, we obtain $T^{\prime}$-consistent indecomposable pp-type $p(\mathbf{x})$, for which $D \Psi=p^{+}(\mathbf{x})$ and $H(p) \in I(T)^{\prime}$.

Next, we consider how close reflection comes to defining a homeomorphism between ${ }_{R} I$ and $I_{R}$. The approach which we propose depends on the existence of generic points of the irreducible closed subsets of ${ }_{R} I\left(I_{R}\right)$. Recall that an irreducible closed set $I$ is one which cannot be written as a union of proper closed subsets and that a generic point $U \in I$ is an element whose closure is $I$.

Lemma 4.7. If $I \subseteq{ }_{R} I\left(I_{R}\right)$ is an irreducible closed set so that the relative topology on I has a countable basis, then I has a generic point.

Proof. Let $\left\{O_{n} \mid n<\omega\right\}$ be an enumeration of the nonempty members of the basis. We define a sequence $\left\{\left(\varphi_{n} / \psi_{n}\right) \mid n<\omega\right\}$ of nonempty open subsets of $I$ such that $\left(\varphi_{n} / \psi_{n}\right) \subseteq O_{n}$ and $\psi_{n} \subseteq \psi_{n+1} \subseteq \varphi_{n+1} \subseteq \varphi_{n}$ with all ppfs in one variable. Assume, for ease of argument, that $O_{0}={ }_{R} I\left(I_{R}\right)$ and so we can take $\varphi_{0} \Leftrightarrow x=x$ and $\psi_{0} \Leftrightarrow x=0$. Given $\varphi_{n} / \psi_{n}$, we know that $\left(\varphi_{n} / \psi_{n}\right) \cap O_{n+1} \neq \varnothing$ since $I$ is irreducible. Let $U \in\left(\varphi_{n} / \psi_{n}\right) \cap O_{n+1}$ and take $c \in \varphi_{n}(U) \backslash \psi_{n}(U)$. By [Z, Theorem 4.9], there is a $\varphi_{n+1} / \psi_{n+1} \in \operatorname{tp}(c)$ such that $\psi_{n} \subseteq \psi_{n+1} \subseteq \varphi_{n+1} \subseteq$ $\varphi_{n}$ and $\left(\varphi_{n+1} / \psi_{n+1}\right) \subseteq O_{n+1}$.

Let $\Phi$ be the filter generated by the $\left\{\varphi_{n} \mid n<\omega\right\}$ and $\Psi$ the ideal generated by the $\left\{\psi_{n} \mid n<\omega\right\}$. Applying [P, Lemma 4.33] at $T(I)$, gives us a $T(I)$ consistent indecomposable pp-type $p$ for which $\vDash p^{+}(x) \rightarrow \Phi(x)$ and $\Psi \subseteq$ $p^{-}(x)$. It is now clear that $U=H(p)$ is a generic point of $I$, since $U \in$ $\left(\varphi_{n} / \psi_{n}\right) \subseteq O_{n}$ for each $n<\omega$.

Definition 4.8. For $U, V \in{ }_{R} I\left(I_{R}\right)$, set $U \approx V$ if $I(U)=I(V)$. Denote by ${ }_{R} I / \approx\left(I_{R} / \approx\right)$ the quotient space endowed with the quotient topology.

Corollary 4.9. If $R$ is countable, then ${ }_{R} I / \approx$ and $I_{R} / \approx$ are homeomorphic.

Proof. Let $U \in{ }_{R} I\left(I_{R}\right)$ represent a class in ${ }_{R} I / \approx\left(I_{R} / \approx\right) . I(U)$ is an irreducible closed set. It follows from Proposition 4.4 that $I(U)^{\prime}$ is irreducible as well. Since $R$ is countable, ${ }_{R} I\left(I_{R}\right)$ has a countable basis. By Lemma 4.6, $I(U)^{\prime}$ has a generic point $V$ and if $V_{0}$ is another such point, then $V \approx V_{0}$, so that the function $h:{ }_{R} I \rightarrow I_{R}\left(h^{\prime}: I_{R} \rightarrow{ }_{R} I\right)$ defined by $h(U)=V$ induces a well-defined function from ${ }_{R} I / \approx\left(I_{R} / \approx\right)$ to $I_{R} / \approx\left({ }_{R} I / \approx\right)$. Since $h^{-1}(I / \approx)$ $=I^{\prime} / \approx, h$ is continuous (and similarly for $\left.h^{\prime}\right)$. $h \circ h^{\prime}$ and $h^{\prime} \circ h$ induce the identity maps on ${ }_{R} I / \approx$ and $I_{R} / \approx$ respectively and the result follows.

Let $U \in{ }_{R} I\left(I_{R}\right)$ and let $I=I(U)$. Suppose that $U$ realizes an $I$-minimal i.e., Latt $(I)$-minimal, pair $\varphi / \psi$. In other words, $\varphi(U) / \psi(U) \neq 0$ and there is no $\sigma$ which, as far as $I$ is concerned, lies strictly between $\psi$ and $\varphi$. Call such a $U$ reflexive. By Proposition 4.5, $D \psi / D \varphi$ is an $I^{\prime}$-minimal pair. Let $D U \in I^{\prime}$ realize $D \psi / D \varphi$. By [Z, Lemma 7.10], $D U$ is unique and by Proposition 4.4 it does not depend on our choice of the minimal pair $\varphi / \psi$. We say that $D U$ is 
the dual of $U$. Occasionally, we shall use the fact (which follows quickly from [Z, Theorem 4.9]) that if $U$ realizes an $I$-minimal pair, then for every a $\in U$ there is an $I$-minimal pair $\sigma / \tau \in \operatorname{tp}^{+}(\mathbf{a})$ i.e., $\sigma(\mathbf{x}) \in \operatorname{tp}^{+}(\mathbf{a})$ and $\tau(\mathbf{x}) \in \operatorname{tp}^{-}(\mathbf{a})$.

Theorem 4.10. Duality, the map which takes $U$ to $D U$, is a bijection from the set of (isomorphism types of) reflexive pure-injective indecomposable left $R$ modules to the set of (isomorphism types of) reflexive pure-injective indecomposable right $R$-modules and conversely. For a reflexive $U$, we have

(i) $D^{2} U=U$,

(ii) $I(D U)=I(U)^{\prime}$,

(iii) $\operatorname{Latt}(D U) \cong \operatorname{Latt}(U)^{\text {op }}$ via the map which takes $\varphi(U)$ to $D \varphi(D U)=$ $\{\mathbf{c} \in D U \mid \mathbf{c} \otimes \varphi(U)=0$ in $D U \otimes U\}$.

Proof. (i) follows immediately from the definition of $D U$.

(ii) follows as soon as we notice that because $I(U)$ is an irreducible closed set, so is $I(U)^{\prime}$ and $D U$ is a generic point of $I(U)^{\prime}$. So $I(U)^{\prime}=I(D U)$.

(iii) Using Proposition 4.5, everything is immediate except that $D \varphi(D U)=$ $\{c \in D U \mid \mathbf{c} \otimes \varphi(U)=0$ in $D U \otimes U\}$. From Proposition 3.2, we know that $D \varphi(D U) \subseteq\{\mathbf{c} \in D U \mid \mathbf{c} \otimes \varphi(U)=0$ in $D U \otimes U\}$.

To prove the other inclusion, suppose that e $\in D U$ but that e $\notin D \varphi(D U)$. Let $D \tau / D \sigma \in \operatorname{tp}^{+}(\mathbf{e})$ be an $I(D U)$-minimal pair. By [Z, Corollary 4.6], we may choose $D \tau / D \sigma$ so that $D \varphi \subseteq D \sigma \subseteq D \tau$. Now let $D \Phi$ be an ideal maximal with respect to the properties that $D \sigma \in D \Phi$ and $D \Phi \cap \operatorname{tp}^{+}(\mathbf{e})=\varnothing$. Then $\Phi=D D \Phi$ is a filter and, by Lemma 4.6 at $\operatorname{Th}(D U)$, there is an indecomposable type $p(\mathbf{x})$, $H(p) \in I(U)$ such that $\Phi=p^{+}(\mathbf{x})$ and $p^{-}(\mathbf{x}) \supseteq D \mathrm{tp}^{+}(\mathbf{e})$. Let $\vDash p(\mathbf{a})$. Then $H(\mathbf{a}) \in I(U)$ and $\sigma / \tau \in \operatorname{tp}^{+}(\mathbf{a})$ so $H(\mathbf{a}) \in(\sigma / \tau)$. This forces $H(\mathbf{a})=U$ and as $\sigma(U) \subseteq \varphi(U)$, we have that $\mathbf{a} \in \varphi(U)$. But, using Proposition 3.2, $\mathbf{e} \otimes \mathbf{a} \neq 0$ in $D U \otimes U$ because if $\psi \in \operatorname{tp}^{+}(\mathbf{a})=\Phi$, then $D \psi \notin \operatorname{tp}^{+}(\mathbf{e})$, by the definition of $D \Phi$. Thus e $\notin\{\mathrm{c} \in D U \mid \mathbf{c} \otimes \varphi(U)=0$ in $D U \otimes U\}$ and the second inclusion is proved.

\section{Problem 4.11. Compute $D U \otimes_{R} U$.}

Since we have associated to the left module $U$ a right module $D U$, Problem 4.11 is a natural one to pose. We shall see in the sequel that it is easier to compute $D U \otimes_{R} U$ en route to understanding $D U$.

If $m-\operatorname{dim}(I)<\infty$ and $U \in I$, then $m-\operatorname{dim}(I(U))<\infty$ and hence $U$ realizes an $I(U)$-minimal pair and is, therefore, reflexive. As $m-\operatorname{dim}\left(I^{\prime}\right)=m$-dim( $(I)$, the same considerations show that each $V \in I^{\prime}$ is reflexive (cf. Proposition 0.7). It follows that reflection is a homeomorphism between $I$ and $I^{\prime}$.

\section{DEDEKIND DOMAINS}

In this section, we determine the reflection of a pure-injective indecomposable over a Dedekind domain. We shall rely heavily upon the consequence of Theorem 4.10 that for a reflexive $U, U \vDash \psi(\mathbf{x}) \rightarrow \varphi(\mathbf{x})$ iff $D U \vDash D \varphi(\mathbf{x}) \rightarrow D \psi(\mathbf{x})$. Recall that a pure-injective indecomposable $U$ has a local endomorphism ring. Thus when $U$ is over a commutative ring $R$, there is a prime ideal $\wp \subseteq R$, $\wp=m \cap R, m \subseteq$ End $_{R} U$ the maximal ideal, such that $U$ is a module over $R_{\wp}$, the localization of $R$ at $\wp$. In the following lemma, assume that $U$ is a reflexive pure-injective indecomposable. 
Lemma 5.1. (i) $\operatorname{ann}(U)=\operatorname{ann}(D U)$.

(ii) $r U=U$ iff $D U[r]=0(M[r]=\{m \in M \mid r m=0(m r=0)\})$.

(iii) If $R$ is commutative and $U$ is an $R_{\wp}$-module, then so is $D U$.

Proof. (i) If $r \in \operatorname{ann}(U)$, then $U \vDash r x=0 \leftrightarrow x=x$. Applying $D$ shows that $D U \vDash r \mid x \leftrightarrow x=0$ i.e., that $D U r=0$. The other inclusion is similar.

(ii) $r U=U$ iff $U \vDash r \mid x \leftrightarrow x=x$ iff $D U \vDash x r=0 \leftrightarrow x=0$ iff $D U[r]=0$.

(iii) follows immediately from (i) and (ii).

Thus if $R$ is a commutative (von Neumann) regular ring, then $U \cong D U$ for each reflexive $U \in{ }_{R} I$. This is so because both $U$ and $D U$ are indecomposable modules over some field of the form $R_{\wp}$.

Ziegler [Z, Lemma 5.1] has determined all of the pure-injective indecomposables over a Dedekind domain, $R$. They are

(i) $Q$, the field of fractions of $R$ and for each nonzero prime ideal of $R$, $\wp$

(ii) $R / \wp^{n}$ for $n \geq 1$,

(iii) $\widetilde{R}_{\wp}$ the $\wp$-adic completion of the localization of $R$ at $\wp$, and

(iv) $Q / R_{\wp}$.

As a matter of fact, $m-\operatorname{dim}\left({ }_{R} I\right)=2$, so that all of these are reflexive. We proceed to determine their reflections.

(i) As $Q$ is the only pure-injective indecomposable over $R_{\{0\}}$, Lemma 5.1(iii) implies that $D Q=Q$.

(ii) $D\left(R / \wp^{n}\right)$ must be a pure-injective indecomposable over $R_{\wp}$ whose annihilator is $\wp^{n}$ so it has no choice but to be itself.

(iii) and (iv) Both $\widetilde{R}_{\wp}$ and $Q / R_{\wp}$ are faithful $R_{\wp}$-modules and $\widetilde{R}_{\wp}$ is torsionfree, but not divisible and $Q / R_{\wp}$ is divisible, but not torsion-free. It follows then from Lemma 5.1 (ii) that $D \widetilde{R}_{\wp}=Q / R_{\wp}$ and hence that $D\left(Q / R_{\wp}\right)=\widetilde{R}_{\wp}$.

\section{LocAlizATION AT A CLOSED SET}

Mike Prest has introduced [P, Chapter 8] the notion of localization at a closed set $I$. Localization is tantamount to doing things modulo $T(I)$. Although seemingly innocuous, it will be useful for us in making some key observations. In particular, we shall show that a notion of duality exists for complete theories of modules.

As $\mathrm{Ob}(R$-Mod $)=\left\{M \mid I(M) \subseteq{ }_{R} I\right\}$, we define its localization at $I, I$-Mod, to be the full subcategory of $R$-Mod for which $\mathrm{Ob}(I$-Mod $)=\{M \mid I(M) \subseteq I\}$. In continuity with the definition of $(R \text {-Mod })^{\text {eq }},(I-M o d)^{\text {eq }}$ will be the category of functors from $I$-Mod to $\mathrm{Ab}$ expressible as pp-pairs in $\operatorname{Latt}(I)$.

Definition 6.1. $(I \text {-Mod })^{\text {eq }}\left(\left(\text { Mod- } I^{\prime}\right)^{\text {eq }}\right)$, the localization of $(R \text {-Mod })^{\text {eq }}$ $\left((R \text {-Mod })^{\text {eq }}\right)$ at $I\left(I^{\prime}\right)$ is defined as follows.

(a) $\mathrm{Ob}\left((I \text {-Mod })^{\text {eq }}\right) \mathrm{Ob}\left(\left(\operatorname{Mod}-I^{\prime}\right)^{\text {eq }}\right)=\left\{\varphi / \psi \mid I\left(I^{\prime}\right) \vDash \psi \rightarrow \varphi\right.$ and both formulae are ppfs in $\left.{ }_{R} L\left(L_{R}\right)\right\}$ modulo $I\left(I^{\prime}\right)$ i.e., we identify $\varphi / \psi$ and $\varphi_{0} / \psi_{0}$ if $I\left(I^{\prime}\right) \vDash \varphi \leftrightarrow \varphi_{0}$ and $I\left(I^{\prime}\right) \vDash \psi \leftrightarrow \psi_{0}$.

(b) $\rho \in \operatorname{Mor}_{I}(\varphi / \psi, \sigma / \tau)\left(\operatorname{Mor}_{I^{\prime}}(\psi / \varphi, \tau / \sigma)\right)$ iff there is a ppf $\rho(\mathbf{x}, \mathbf{y}) \in$ ${ }_{R} L\left(L_{R}\right)$ such that

(i) $I \vDash \varphi(\mathbf{x}) \rightarrow \exists \mathbf{y} \rho(\mathbf{x}, \mathbf{y})\left(I^{\prime} \vDash \tau(\mathbf{y}) \rightarrow \exists \mathbf{x} \rho(\mathbf{x}, \mathbf{y})\right)$,

(ii) $I \vDash \psi(\mathbf{x}) \rightarrow \rho(\mathbf{x}, 0)\left(I^{\prime} \vDash \sigma(\mathbf{y}) \rightarrow \rho(0, \mathbf{y})\right)$, 
(iii) $I \vDash \exists \mathbf{x} \rho(\mathbf{x}, \mathbf{y}) \rightarrow \sigma(\mathbf{y})\left(I^{\prime} \vDash \exists \mathbf{y} \rho(\mathbf{x}, \mathbf{y}) \rightarrow \psi(\mathbf{x})\right)$,

(iv) $I \vDash \rho(0, \mathbf{y}) \rightarrow \tau(\mathbf{y})\left(I^{\prime} \vDash \rho(\mathbf{x}, 0) \rightarrow \varphi(\mathbf{x})\right)$.

As with $(R \text {-Mod })^{\text {eq }}\left((\text { Mod- } R)^{\text {eq }}\right)$, we identify morphisms which are represented by $I$-equivalent ppfs.

Consider the category $R$-Top (Top- $R$ ) whose objects are the closed subsets of ${ }_{R} I\left(I_{R}\right)$ and such that $\operatorname{Mor}(I, J)=\varnothing$ iff $J \not \subset I$ and if $J \subseteq I$, then $|\operatorname{Mor}(I, J)|=1$. Reflection, taking $I$ to $I^{\prime}$, may be thought of as an isomorphism between $R$-Top and Top- $R$. There is a functor Latt from $R$-Top (Top- $R$ ) to the category of modular lattices which takes a closed set $I$ to $\operatorname{Latt}(I)$, its pp-lattice. If $J \subseteq I$, then there is a natural projection of $\operatorname{Latt}(I)$ onto $\operatorname{Latt}(J)$ which takes the element of $\operatorname{Latt}(I)$ represented by the ppf $\varphi$ to the element of $\operatorname{Latt}(J)$ represented by $\varphi$. This is well defined because $I \vDash \varphi \leftrightarrow \psi$ implies $J \vDash \varphi \leftrightarrow \psi$. Proposition 4.5 states that Latt commutes with reflection.

More generally, $I \vDash|\varphi / \psi|=1$ implies $J \vDash|\varphi / \psi|=1$ when $J \subseteq I$ and this gives rise to a functor from $(I-\mathrm{Mod})^{\mathrm{eq}}$ to $(J \text {-Mod })^{\text {eq }}$ which takes an object of $(I \text {-Mod })^{\text {eq }}$ represented by a given pp-pair into the object of $(J \text {-Mod })^{\text {eq }}$ represented by that same pair and similarly for morphisms. This constitutes a functor ( -Mod) $)^{\text {eq }}\left((\text { Mod- })^{\text {eq }}\right)$ from $R$-Top (Top- $R$ ) to the category of categories. As above, Proposition 4.5 asserts that this functor commutes with reflection. If, in the proof of Theorem 2.9, we replace ${ }_{R} I \vDash$ with $I \vDash$ and $I_{R} \vDash$ with $I^{\prime} \mid=$, we get

Proposition 6.2 (local version of Theorem 2.9). $(I-\mathrm{Mod})^{\mathrm{eq}} \cong\left(\operatorname{Mod}-I^{\prime}\right)^{\mathrm{eq}}$.

In particular, we can localize at $I$ the ring $R(\varphi / \psi)=\operatorname{Mor}(\varphi / \psi, \varphi / \psi)$ to get (definition) $R(\varphi / \psi)^{I}=\operatorname{Mor}_{I}(\varphi / \psi, \varphi / \psi)$. The following is then a consequence of Proposition 6.2.

Corollary 6.3 (local version of Proposition 3.4). $R(\varphi / \psi)^{I} \cong R(D \psi / D \varphi)^{I^{\prime}}$.

If $U$ is a pure-injective indecomposable, then $S=\operatorname{End}_{R} U$ is a local ring. Denote by $\Delta$ the residue division ring of $S$ If $U \in R$-Mod, let $S$ act on $U$ from the right and if $U$ is reflexive, let $S^{\prime}=\operatorname{End}_{R} D U$ act on $D U$ from the left and denote the corresponding division ring by $\Delta^{\prime}$.

Proposition 6.4. $\Delta \cong \Delta^{\prime}$.

Proof. Let $\varphi / \psi$ be a $I(U)$-minimal pair and let $I=I(U)$. To prove the proposition, it is enough, by Corollary 6.3 , to show that $\Delta \cong R(\varphi / \psi)^{I}$ and $\Delta^{\prime} \cong R(D \psi / D \varphi)^{I^{\prime}}$. As in the case of vector spaces, these isomorphisms are not canonical. Since the two assertions have the same proof, we only prove the first. We shall show that $\varphi(U) / \psi(U)$ is a one-dimensional (left) vector space over the division ring $R(\varphi / \psi)^{I}$ and a one-dimensional (right) vector space over $\Delta$. The two actions respect each other $-\Delta \cong R(\varphi / \psi)^{I}$ will follow.

(a) The right action. $\varphi(U) / \psi(U)$ is a right $S$-module. Let a $\in \varphi(U) \backslash \psi(U)$. By [P, Corollary 9.25], $U \vDash \operatorname{pp}-\operatorname{tp}(\mathbf{a}) \leftrightarrow \operatorname{tp}^{+}(\mathbf{a}) \wedge \neg \psi(\mathbf{x})$. So if $f \in S$ is not a unit then [P, Corollary 4.13] implies $U \vDash \psi(\mathbf{a} f)$ and $\varphi(U) / \psi(U)$ is killed by $f$. This means that $\varphi(U) / \psi(U)$ has the structure of a right vector space over $\Delta$. We want to show that it is one dimensional. So let $c \in \varphi(U) \backslash \psi(U)$. If $\sigma \in \operatorname{tp}^{+}(\mathbf{a})$ and $\vDash \sigma \rightarrow \varphi$, then since $\varphi / \psi$ is minimal $\sigma+\psi=\varphi$. Thus $\left\{\mathbf{c}=\mathbf{x}+\mathbf{y}, \psi(\mathbf{y}), \sigma(\mathbf{x}) \mid \sigma(\mathbf{x}) \in \operatorname{tp}^{+}(\mathbf{a})\right\}$ is finitely satisfiable in $U$ and hence, by 
Proposition 0.4 , realized in $U$ by say (b, d). Let $f \in S$ be such that $(\mathbf{a}) f=\mathbf{b}$. Then $U \vDash \psi((\mathbf{a}) f-\mathbf{c})$ and $(\mathbf{a}) f \equiv \mathbf{c}(\bmod \psi(U))$. This means that $\varphi(U) / \psi(U)$ is a one-dimensional vector space over $\Delta$.

(b) The left action. We saw earlier how $\varphi(U) / \psi(U)$ is a left $R(\varphi / \psi)^{I}$ module. Since $\varphi / \psi$ is an $I(U)$-minimal pair, an argument akin to the proof of Schur's Lemma shows that each nonzero $\rho \in R(\varphi / \psi)^{I}$ is an isomorphism and hence that $R(\varphi / \psi)^{I}$ is a division ring. We want to show that $\varphi(U) / \psi(U)$ is one dimensional over $R(\varphi / \psi)^{I}$.

Using the $I$-minimality of $\varphi / \psi$ and the fact that $I=I(U)$, the definition of $R(\varphi / \psi)^{I}=\operatorname{Mor}_{I(U)}(\varphi / \psi, \varphi / \psi)$ indicates that we may identify it with the ppdefinable $Z$-endomorphisms of $\varphi(U) / \psi(U)$. Thus if $\mathbf{a}, \mathbf{c} \in \varphi(U) \backslash \psi(U)$ are such that their images in $\varphi(U) / \psi(U)$ are linearly independent over $R(\varphi / \psi)^{I}$, we would get, by Proposition 3.8, an $f \in S$ that induces a sort endomorphism of $\varphi(U) / \psi(U)$ which fixes $\overline{\mathbf{a}}$ and takes $\overline{\mathbf{c}}$ to 0 . This means that $\vDash \neg \psi(\mathbf{a} f)$ and $\vDash \psi(\mathbf{c} f)$ so that $[\mathrm{P}$, Corollary 4.13] gives the contradiction that $f$ is at the same time a unit as well as a nonunit of $S$.

Corollary 6.5. If $\varphi / \psi$ is a $U$-minimal pair, then

$$
|\varphi(U) / \psi(U)|=|D \psi(D U) / D \varphi(D U)| .
$$

Proof. By the proof of Proposition 6.4,

$$
|\varphi(U) / \psi(U)|=|\Delta|=\left|\Delta^{\prime}\right|=|D \psi(D U) / D \varphi(D U)| .
$$

Let $T$ be a complete theory of left (right) $R$-modules and $I=I(T)$ its closed set. Let $I_{u}$ be the closed set $\{U \in I \mid U$ is $T$-unlimited i.e., $T \oplus \operatorname{Th}(U) \equiv$ $T\}$. This is closed because by [P, Corollary 4.44] any $T$-limited $U$ realizes a $T$-finite pair $\sigma / \tau$ and hence a $T$-minimal pair which isolates $U$ among $I(T)$. In particular, every $U \in I \backslash I_{u}$ is reflexive. If $\varphi / \psi$ is $T$-minimal and isolates $U \in I \backslash I_{u}$, then $|\varphi / \psi|_{T}$ is a power of $|\varphi(U) / \psi(U)|$ and $d_{U}(T)=$ $\log _{|\varphi(U) / \psi(U)|}|\varphi / \psi|_{T}$ is the number of times that $U$ occurs as a direct summand of any pure-injective model of $T$ (cf. [Z, p. 180]). Define the complete theory of right (left) $R$-modules $D T$ as $D T=\operatorname{Th}\left(\left\{\bigoplus(D U)^{d_{U}(T)} \mid U \in I \backslash I_{u}\right\}\left\{\bigoplus V^{\omega} \mid V \in\right.\right.$ $\left.I_{u}^{\prime}\right\}$ ) .

Theorem 6.6. Duality, the map which takes $T$ to $D T$, is a bijection from the class of complete theories of left (right) $R$-modules to the class of complete theories of right (left) $R$-modules. $D T$ is uniquely determined by $T$ via the equation $|\varphi / \psi|_{T}=|D \psi / D \varphi|_{D T}$. If $U$ is reflexive, then $D(\operatorname{Th}(U))=\operatorname{Th}(D U)$.

Proof. From the definition of $D T$, we have that $I(D T)=I(T)^{\prime}$ so that $\operatorname{Latt}(D T)=\operatorname{Latt}(I(D T))=\operatorname{Latt}\left(I(T)^{\prime}\right) \cong \operatorname{Latt}(I(T))^{\text {op }}=\operatorname{Latt}(T)^{\text {op }}$. We split the proof of $|\varphi / \psi|_{T}=|D \psi / D \varphi|_{D T}$ into two cases, depending on whether or not $\varphi / \psi$ has a composition series of pp-definable subgroups (in $T$ ).

(i) If not, then $D \psi / D \varphi$ does not have such a composition series either and we get that $|\varphi / \psi|_{T}=\infty=|D \psi / D \varphi|_{D T}$.

(ii) If $\varphi=\varphi_{0} \supseteq \varphi_{1} \supseteq \cdots \supseteq \varphi_{n+1}=\psi$ is a composition series of $\varphi / \psi$ in $T$, then $D \psi=D \varphi_{n+1} \subseteq D \varphi_{n} \subseteq \cdots \subseteq D \varphi_{1} \subseteq D \varphi_{0}=D \varphi$ is one of $D \psi / D \varphi$ in $D T$. Since $|\varphi / \psi|_{T}=\prod\left\{\left|\varphi_{i} / \varphi_{i+1}\right|_{T} \mid 0 \leq i \leq n\right\}(\bmod \infty)$ and $|D \psi / D \varphi|_{D T}=$ $\prod\left\{\left|D \varphi_{i+1} / D \varphi_{i}\right|_{D T} \mid 0 \leq i \leq n\right\}(\bmod \infty)$ we may assume that $\varphi / \psi$ is a minimal pair. Take $U \in I(T)$ isolated by $\varphi / \psi$. If $U \in I_{u}$, then $(\varphi / \psi) \cap I_{u} \neq \varnothing$ 
and so $(D \psi / D \varphi) \cap I_{u}^{\prime} \neq \varnothing$ and so, by definition of $D T,|\varphi / \psi|_{T}=\infty=$ $|D \psi / D \varphi|_{D T}$. On the other hand, if $U \notin I_{u}$, then $|\varphi / \psi|_{T}<\infty$ and $|\varphi / \psi|_{T}=$ $|\varphi(U) / \psi(U)|^{d_{U}(T)}$. But $|D \psi / D \varphi|_{D T}=|D \psi(D U) / D \varphi(D U)|^{d_{D U}(D T)}$ and we defined $D T$ so that $d_{D U}(D T)=d_{U}(T)$. By Corollary 6.5, $|\varphi(U) / \psi(U)|=$ $|D \psi(D U) / D \varphi(D U)|$ and so $|\varphi / \psi|_{T}=|D \psi / D \varphi|_{D T}$ follows.

For $U$ reflexive, $D(\operatorname{Th}(U))=\operatorname{Th}(D U)$ follows from computing the respective BGM-invariants and applying the first part of the theorem.

Let ${ }_{R} N \vDash T$ and $N^{\prime} \vDash D T$ and $I=I(T)$. Then $\varphi / \psi(N)$ is a left $R(\varphi / \psi)^{I}$ module. Let $A=R(\varphi / \psi)^{I}$. We also know that $(D \psi / D \varphi)\left(N^{\prime}\right)$ is a right $A$-module, from Corollary 6.3.

Proposition 6.7. Under the above conditions,

$$
D\left(\operatorname{Th}\left({ }_{A} \varphi / \psi(N)\right)\right)=\operatorname{Th}\left((D \psi / D \varphi)\left(N^{\prime}\right)_{A}\right) .
$$

Proof. We may assume that $\varphi / \psi$ is a pair of ppfs in one variable.

Lemma 6.8. For each ${ }_{A} \sigma(\mathbf{x}) \in{ }_{A} L, l(\mathbf{x})=n$, there is a ppf ${ }_{R} \sigma(\mathbf{x}) \in_{R} L$ with $\vDash \psi^{n}(\mathbf{x}) \rightarrow{ }_{R} \sigma(\mathbf{x})$ and $\vDash{ }_{R} \sigma(\mathbf{x}) \rightarrow \varphi^{n}(\mathbf{x})$ such that ${ }_{A} \sigma\left({ }_{A} \varphi / \psi(N)\right)={ }_{R} \sigma(N) / \psi(N)$ and $D\left({ }_{A} \sigma\right)\left(D \psi / D \varphi\left(N^{\prime}\right)_{A}\right)=D\left({ }_{R} \sigma\right)\left(N^{\prime}\right) / D \varphi\left(N^{\prime}\right)$.

Once this lemma is proved, the proposition follows from Theorem 6.6 and the fact that for every pp-pair ${ }_{A} \sigma !{ }_{A} \tau \in{ }_{A} L$ in one variable $\left|\left({ }_{A} \sigma /{ }_{A} \tau\right)\left({ }_{A} \varphi / \psi(N)\right)\right|$ $=\left|{ }_{R} \sigma(N) / \psi(N) /_{R} \tau(N) / \psi(N)\right|=\left|{ }_{R} \sigma /{ }_{R} \tau(N)\right|=\left|D\left({ }_{R} \tau\right) / D\left({ }_{R} \sigma\right)\left(N^{\prime}\right)\right|=$ $\left|D\left({ }_{R} \tau\right)\left(N^{\prime}\right) / D \varphi\left(N^{\prime}\right) / D\left({ }_{R} \sigma\right)\left(N^{\prime}\right) / D \varphi\left(N^{\prime}\right)\right|=\left|D\left({ }_{A} \tau\right) / D\left({ }_{A} \sigma\right)\left(D \psi / D \varphi\left(N^{\prime}\right)_{A}\right)\right|$.

Proof of Lemma 6.8. Let ${ }_{A} \sigma(\mathbf{x}) \Leftrightarrow \exists \mathbf{y}(H J)\left(\begin{array}{l}\mathbf{x} \\ \mathbf{y}\end{array}\right)=0$ with $H$ an $m \times n$ matrix over $A$ and $J$ and $m \times k$ matrix over $A$. Thus $l(\mathbf{x})=n$ and $l(\mathbf{y})=k$. Note that $(H J) \in \operatorname{Mor}\left((\varphi / \psi)^{n+k},(\varphi / \psi)^{m}\right)$ and that if we denote by $(H J)\left(\mathbf{x}^{\wedge} \mathbf{y}, \mathbf{z}\right)$ a ppf representing $(H J)$, then the dual $D(H J)\left(\mathbf{x}^{\wedge} \mathbf{y}, \mathbf{z}\right)$ represents (cf. Example 2.5). $(H J) \in \operatorname{Mor}\left((D \psi / D \varphi)^{n+k},(D \psi / D \varphi)^{m}\right)$, the morphism which acts from the right as $(H J):(D \psi / D \varphi)^{m} \rightarrow(D \psi / D \varphi)^{n+k}$. Let $\rho(\mathbf{x}, \mathbf{y}) \Leftrightarrow$ $\exists \mathbf{z}(H J)\left(\mathbf{x}^{\wedge} \mathbf{y}, \mathbf{z}\right) \wedge\left(\psi^{m}\right)(\mathbf{z})$ and let ${ }_{R} \sigma(\mathbf{x}) \Leftrightarrow \exists \mathbf{y} \rho(\mathbf{x}, \mathbf{y})$; then ${ }_{R} \sigma(\mathbf{x})$ induces ${ }_{A} \sigma(\mathbf{x})$ on $\varphi / \psi$. By Proposition 2.7, we get $I^{\prime} \vDash D \rho\left(\mathbf{x}^{\wedge} \mathbf{y}\right) \leftrightarrow \exists \mathbf{z} D(H J)\left(\mathbf{x}^{\wedge} \mathbf{y}, \mathbf{z}\right) \wedge$ $D\left(\psi^{m}\right)(\mathbf{z})$ and so it follows that $I^{\prime} \vDash D \rho(\mathbf{x}, \mathbf{y}) \leftrightarrow \exists \mathbf{z} D(H J)\left(\mathbf{x}^{\wedge}-\mathbf{y}, \mathbf{z}\right) \wedge$ $D\left(\psi^{m}\right)(\mathbf{z})$. Applying to $(H J)\left(\mathbf{x}^{\wedge} \mathbf{y}, \mathbf{z}\right)$ condition (i) of the definition of a morphism, we get $I \vDash{ }_{R} \sigma(\mathbf{x}) \leftrightarrow \exists \mathbf{y} \rho(\mathbf{x}, \mathbf{y}) \wedge \varphi^{k}(\mathbf{y})$. By Proposition 2.7 $I^{\prime} \vDash D\left({ }_{R} \sigma\right)(\mathbf{x}) \leftrightarrow \exists \mathbf{y} D \rho(\mathbf{x}, \mathbf{y}) \wedge D\left(\varphi^{k}\right)(\mathbf{y})$ so that

$$
I^{\prime} \vDash\left({ }_{R} \sigma\right)(\mathbf{x}) \leftrightarrow \exists \mathbf{y}, \mathbf{z} D(H J)\left(\mathbf{x}^{\wedge}-\mathbf{y}, \mathbf{z}\right) \wedge D\left(\psi^{m}\right)(\mathbf{z}) \wedge D\left(\varphi^{k}\right)(\mathbf{y})
$$

follows and hence $I^{\prime} \vDash D\left({ }_{R} \sigma\right)(\mathbf{x}) \leftrightarrow \exists \mathbf{y}, \mathbf{z} D(H J)^{\prime}\left(\mathbf{x}^{\wedge} \mathbf{y}, \mathbf{z}\right) \wedge D\left(\psi^{m}\right)(\mathbf{z}) \wedge D\left(\varphi^{k}\right)(\mathbf{y})$. But it is clear that $D\left({ }_{R} \sigma\right)(\mathbf{x})$ induces on $(D \psi / D \varphi)^{n}\left(N^{\prime}\right)$ the subgroup defined by $\exists \mathbf{z} \mathbf{z}(H J)=\left(\begin{array}{l}\mathbf{x} \\ \mathbf{y}\end{array}\right) \wedge \mathbf{y}=0$ i.e., the subgroup defined by $\exists \mathbf{z} \mathbf{z} H=\mathbf{z} \wedge \mathbf{z} J=0$ i.e., the subgroup defined by $D\left({ }_{A} \sigma\right)(\mathbf{x})$.

\section{STRONGLY MINIMAL INDECOMPOSABLES}

As an application of Theorem 6.6 , this section is devoted to the example of strongly minimal modules. Recall that an infinite module $M$ is strongly minimal if every proper pp-definable subgroup $\varphi(M)$ ( $\varphi$ in one variable) of 
$M$ is finite. All such modules are pure injective [P, Theorem 3.1] and one can readily verify that in this case $m$ - $\operatorname{dim}(M) \leq 1$. Thus every strongly minimal indecomposable module $U$ is reflexive and so, in particular, are all $V \in I(U)$, although $I(U)$ is not very big, as we shall see.

Note that if $U$ is strongly minimal, then for each $r \notin \operatorname{ann}(U), r U=U$ so that $R / \operatorname{ann}(U)$ has no zero divisors. We separate our analysis into two cases, depending on $m$-dim $(U)$.

(i) If $m-\operatorname{dim}(U)=1$, we may endow $D U$ with a topology which makes it a compact (Hausdorff) topological module. In this case, $U$ has arbitrarily large finite pp-definable subgroups and $I(U)$ contains only one point apart from $U$. This point, to which we shall refer as $\Delta$, is called the unlimited indecomposable. $\Delta$ is not isolated in $I(U)$ and there is a unique nonzero type $p(x)$ for which $\Delta=H(p)$. This type $p(x)$ is called the unlimited type and it is determined by the equation $p^{-}(x)=\{\psi(x)|| \psi(U) \mid$ is finite $\} . \Delta$ is thus pp-simple i.e., contains no nontrivial pp-definable subgroups, and belongs to that class of strongly minimal indecomposables considered in (ii).

Let $D U$ be the reflection of $U$. By Corollary 6.5, every nonzero definable subgroup of $D U$ is of finite index in $D U$. As $I(U)$ and $I(D U)$ are homeomorphic, $I(D U)$ also has a unique nonisolated point, $D \Delta$, the dual of $\Delta$. $D \Delta$ is also called the unlimited indecomposable. As above, $D \Delta$ is pp-simple and there is a unique nonzero pp-type $D p(x)$, the unlimited type, for which $D \Delta=H(D p)$. $D p(x)$ is determined by $D p^{+}(x)=\{\varphi(x) \mid[D U: \varphi(D U)]$ is finite\}.

Consider the topology on $(D U,+)$ whose basis of (clopen) neighborhoods of the identity is given by the nonzero pp-definable subgroups of $D U . D U$ is Hausdorff since it omits $D p(x)$-so if $a \in \bigcap\{\varphi(D U) \mid[D U: \varphi(D U)]$ is finite $\}$ then $a=0$. + is continuous by definition and for every $r \in R$ and ppf $\varphi(x)$ for which $[D U: \varphi(D U)]$ is finite we have that $r^{-1}(\varphi(D U))$ is a subgroup of $D U$ of finite index defined by the ppf $\varphi(x r) . D U$ is thus a topological module.

We claim that $D U$ is compact. For let $D U=\bigcup\left\{\varphi_{\alpha}(D U)+c_{\alpha} \mid \alpha<\kappa\right\}$ be a cover consisting of basis neighborhoods no finite subset of which covers $D U$. Then $\left\{\neg \varphi_{\alpha}\left(x-c_{\alpha}\right) \mid \alpha<\kappa\right\}$ is consistent and realized by $a \in M$, some pure-injective extension of $D U$. By [Z, Theorem 9.1], $M=D U \oplus D \Lambda^{\lambda}$ so $a=a_{1}+a_{2}$ with $a_{1} \in D U$ and $a_{2} \in D \Delta^{\lambda}$. But then $M \vDash \varphi_{\alpha}\left(a_{2}\right)$ for each $\alpha<\kappa$ and it follows that $a_{1}$ realizes $\left\{\neg \varphi_{\alpha}\left(x-c_{\alpha}\right) \mid \alpha<\kappa\right\}$ as well, contradicting the assumption that $\bigcup\left\{\varphi_{\alpha}(D U)+c_{\alpha} \mid \alpha<\kappa\right\}$ be a cover of $D U$.

(ii) If $U$ is pp-simple, then there is a ring homomorphism $R \rightarrow \Delta$ of $R$ into a division ring $\Delta$ such that ${ }_{R} U \cong{ }_{R} \Delta$ and $D U \cong \Delta_{R}$.

First we note that if $U$ is strongly minimal and $m-\operatorname{dim}(U)=0$, then $U$ is pp-simple. This is so because if $0 \subseteq \varphi_{1}(U) \subseteq \cdots \subseteq \varphi_{n-1}(U) \subseteq U$ is a pp-composition series, then, as in the proof of Corollary 6.5, all the quotients $\varphi_{k+1}(U) / \varphi_{k}(U)$ have the same cardinality. This must be infinite and so by the definition of strongly minimal, $U$ must be pp-simple. Conversely, any infinite pp-simple indecomposable is strongly minimal. Thus the only other indecomposables which we take into account when considering pp-simple ones are the finite pp-simple indecomposables.

As in the proof of Proposition 6.4, $U$ is a one-dimensional (left) vector space over the division ring $\Delta=R(\varphi / \psi)^{I}$. There is a ring homomorphism $\alpha: R \rightarrow \Delta$ 
which sends $r \in R$ into the action of $r$ on $\Delta$ and so we see that $R / \operatorname{ann}(U)$ imbeds into $\Delta$, making $\Delta$ a left $R$-module. Then ${ }_{R} U \cong{ }_{R} \Delta$. Using a similar argument for $D U$ yields $D U_{R} \cong D \Delta_{R}$ and our claim follows from Proposition 6.4 .

\section{LOCAL PURITY}

To test a homomorphism ${ }_{R} N \stackrel{f}{\longrightarrow}{ }_{R} M$ for purity, it is enough, according to Proposition 0.3 , to tensor $f$ against each finitely presented $K \in \operatorname{Mod}-R$. Another class of test modules is provided by the next result. The method of proof brings together many elements of this paper. We shall have occasion to use it more than once.

Proposition 8.1. ${ }_{R} N \stackrel{f}{\longrightarrow}{ }_{R} M$ is pure iff $U \otimes_{R} N \stackrel{1 \otimes f}{\longrightarrow} U \otimes_{R} M$ is injective for each $U \in I_{R}$.

Proof. Left to right is trivial, so assume that the right-hand side holds. Let $\mathbf{a} \in N$ and suppose that $M \vDash \varphi(\mathbf{a} f)$. We must show $N \vDash \varphi(\mathbf{a})$. Suppose not. Let $D \Psi(\mathbf{x})=\operatorname{tp}^{+}(\mathbf{a})$ and let $D \Phi$ be an ideal of ppfs, maximal with respect to the properties that $\varphi \in D \Phi$ and $D \Phi \cap D \Psi=\varnothing$. By Lemma 4.1, there is an indecomposable type $p(\mathbf{x})$ such that $\vDash p^{+}(\mathbf{x}) \leftrightarrow \Phi(\mathbf{x})$ and $\Psi \subseteq p^{-}(\mathbf{x})$. Let $\vDash p(\mathbf{c})$ and let $U=H(\mathbf{c})$ be the indecomposable hull. Proposition 3.2 implies that $\mathbf{c} \otimes \mathbf{a} \neq 0$ in $U \otimes N$ because for every $\psi(\mathbf{x})$ for which $N \vDash \psi(\mathbf{a})$ we have that $U \vDash \neg D \psi(\mathbf{c})$. But $\mathbf{c} \otimes \mathbf{a} f=0$ in $U \otimes M$ since $M \vDash \varphi(\mathbf{a} f)$ and $U \vDash D \varphi(\mathbf{c})$. This contradicts the injectivity of $1 \otimes f$.

For $I \subseteq{ }_{R} I$, a closed set, we shall say that the homomorphism ${ }_{R} N \stackrel{f}{\longrightarrow}{ }_{R} M$ is $I$-pure iff $U \otimes_{R} N \stackrel{1 \otimes f}{\longrightarrow} U \otimes_{R} M$ is injective for each $U \in I^{\prime}$. Note that we have not placed on $f$ the requirement of being injective. We shall also need the following

Definition 8.2. If $\Phi(\mathbf{x})$ is a filter of ppfs $\in_{R} L$ and $I \subseteq{ }_{R} I$ is a closed set, then the localization of $\Phi$ at $I$ is $\Phi^{I}(\mathbf{x})=\{\psi(\mathbf{x}) \mid$ there is a $\varphi(\mathbf{x}) \in \Phi$ and $I \vDash \varphi \rightarrow \psi\}$.

Proposition 8.3. ${ }_{R} N \stackrel{f}{\longrightarrow}{ }_{R} M$ is I-pure iff $\operatorname{tp}^{+}(\mathbf{a} f) \subseteq \operatorname{tp}^{+}(\mathbf{a})^{I}$ for each $\mathbf{a} \in N$.

Proof. $(\Rightarrow)$ Suppose to the contrary that $M \vDash \psi(\mathbf{a} f)$ and $\psi \notin \Phi(x)=\operatorname{tp}^{+}(\mathbf{a})^{I}$ for some $\mathbf{a} \in N$. Thus for each $\varphi \in \operatorname{tp}^{+}(\mathbf{a}), I \vDash|\varphi / \psi|>1$. Let $\Psi(\mathbf{x})$ be an ideal of ppfs maximal with the properties $\psi \in \Psi$ and $\Phi \cup\{\neg \sigma \mid \sigma \in \Psi\}$ is $T(I)$ consistent. Then Lemma 4.6 at $T(I)$ implies that $\vDash D \Psi(\mathbf{x}) \leftrightarrow p^{+}(\mathbf{x})$ for some indecomposable type $p(\mathbf{x}), H(p) \in I^{\prime}$. Let $\vDash p(\mathbf{c})$ and let $U=H(\mathbf{c}) \in I^{\prime}$. Then, using Proposition 3.2, $\mathbf{c} \otimes \mathbf{a} \neq 0$ in $U \otimes N$, but $\mathbf{c} \otimes \mathbf{a} f=0$ in $U \otimes M$, contradicting the $I$-purity of ${ }_{R} N \stackrel{f}{\longrightarrow}{ }_{R} M$.

$(\Leftarrow)$ Suppose that $U \in I^{\prime}, \mathbf{a} \in M$ and $\mathbf{c} \in U$, and $\mathbf{c} \otimes \mathbf{a} f=0$ in $U \otimes M$. By Proposition 3.2, there is a $\psi \in \operatorname{tp}^{+}(\mathbf{a} f)$ and $D \psi \in \operatorname{tp}^{+}(\mathbf{c})$. Since $\operatorname{tp}^{+}(\mathbf{a} f) \subseteq$ $\operatorname{tp}^{+}(\mathbf{a})^{I}$, there is a $\varphi \in \operatorname{tp}^{+}(\mathbf{a})$ such that $I \vDash \varphi \rightarrow \psi$. Then $I^{\prime} \vDash D \psi \rightarrow D \varphi$ and so $D \varphi \in \operatorname{tp}^{+}(\mathbf{c})$. But then, Proposition 3.2 implies that $\mathbf{a} \otimes \mathbf{c}=0$. 
Corollary 8.4 (local version of Proposition 8.1) ${ }_{R} N \stackrel{f}{\longrightarrow}{ }_{R} M$ is pure iff it is $I\left(\operatorname{Th}\left({ }_{R} N\right)\right)$-pure.

Proof. Let $I=I\left(\operatorname{Th}\left({ }_{R} N\right)\right)$. One direction is clear, so take ${ }_{R} N \stackrel{f}{\longrightarrow}{ }_{R} M$ to be $I$-pure. By Proposition 8.3, $\operatorname{tp}^{+}(\mathbf{a} f) \subseteq \operatorname{tp}^{+}(\mathbf{a})^{I}$ for each $\mathbf{a} \in N$. But if $\varphi \in \operatorname{tp}^{+}(\mathbf{a})$ and $I \vDash \varphi \rightarrow \psi$, then $\psi \in \operatorname{tp}^{+}(\mathbf{a})$. Hence $\operatorname{tp}^{+}(\mathbf{a})=\operatorname{tp}^{+}(\mathbf{a})^{I}$. Since $\operatorname{tp}^{+}(\mathbf{a} f) \supseteq \operatorname{tp}^{+}(\mathbf{a})$ is always true, we get that $\operatorname{tp}^{+}(\mathbf{a} f)=\operatorname{tp}^{+}(\mathbf{a})$ and hence that $f$ is pure.

Thus to test ${ }_{R} N \stackrel{f}{\longrightarrow}{ }_{R} M$ for purity, it is enough to tensor this homomorphism against the pure-injective indecomposables which live in the reflection of $I(\operatorname{Th}(N))$. In a similar vein, we can define a homomorphism ${ }_{R} N \stackrel{f}{\longrightarrow}{ }_{R} M$ to be $U$-pure, for a reflexive $U$ if $D U \otimes_{R} N \stackrel{1 \otimes f}{\longrightarrow} D U \otimes_{R} M$ is injective. In this case, we also say that ${ }_{R} N$ is $U$-pure injective if every $U$-pure homomorphism ${ }_{R} N \stackrel{f}{\longrightarrow}{ }_{R} M$ is a split imbedding. There is a canonical way of constructing $U$-pure-injective modules.

Example 8.5. Let $S$ be a ring making ${ }_{S} D U_{R}$ an $S$-R-bimodule and let ${ }_{S} E$ be an injective left $S$-module. Then we claim that ${ }_{R} \operatorname{Hom}_{S}\left({ }_{S} D U,{ }_{S} E\right)$ is $U$-pureinjective. For suppose that ${ }_{R} \operatorname{Hom}_{S}\left({ }_{S} D U,{ }_{S} E\right) \stackrel{f}{\longrightarrow}{ }_{R} M$ is $U$-pure. Then

$$
0 \rightarrow{ }_{S} D U \otimes \operatorname{Hom}_{S}\left({ }_{s} D U,{ }_{S} E\right) \stackrel{f}{\longrightarrow}{ }_{S} D U \otimes M
$$

is exact. Since ${ }_{S} E$ is injective,

$$
\operatorname{Hom}\left({ }_{s} D U \otimes M,{ }_{s} E\right) \rightarrow \operatorname{Hom}\left({ }_{s} D U \otimes \operatorname{Hom}_{S}\left({ }_{s} D U,{ }_{s} E\right),{ }_{s} E\right) \rightarrow 0
$$

is exact. But his is tantamount to the exactness of

$\operatorname{Hom}(M, \operatorname{Hom}(D U, E)) \rightarrow \operatorname{Hom}\left(\operatorname{Hom}_{S}\left({ }_{s} D U,{ }_{s} E\right), \operatorname{Hom}_{S}\left({ }_{s} D U,{ }_{s} E\right)\right) \rightarrow 0$.

If we choose a preimage of $1 \in \operatorname{End}\left(\operatorname{Hom}_{S}\left({ }_{S} D U,{ }_{S} E\right)\right), g$ say, then $f g$ is the identity on $\operatorname{Hom}_{S}\left(s D U,{ }_{s} E\right)$ and so $f$ is a split imbedding.

To prove a pointwise version of Corollary 8.4, we need the following lemma, whose proof appears as a proof of [P, Corollary 9.25] in an early draft of [P].

Lemma 8.6. Suppose that $U=H(p), \varphi / \psi \in p$ is a $U$-minimal pair. Then $\vDash p(x) \leftrightarrow p^{+}(x) \cup\{\neg \sigma(x) \mid I(U) \vDash \sigma \rightarrow \psi\}$.

Proof. It is enough to show that $\vDash p^{+}(x) \cup\{\neg \sigma(x) \mid I \vDash \sigma \rightarrow \psi\} \rightarrow p^{-}(x)$. So let $\delta \in p^{-}(x)$. By [Z, Theorem 4.4], there is a $\tau \in p^{+}(x)$ such that $\vDash \tau(x) \rightarrow \varphi(x)$ and $(\tau \cap \delta)+(\tau \cap \psi) \in p^{-}(x)$. Now $\tau \cap \psi \subseteq \tau \cap \psi+\tau \cap \delta \subseteq \tau$ and $\tau / \tau \cap \psi$ is a $U$-minimal pair, so it must be that $U \notin(\tau \cap \psi+\tau \cap \delta / \tau \cap \psi)=$ $(\tau \cap \delta / \tau \cap \delta \cap \psi)=(\tau \cap \delta+\psi / \psi)$ and $\vDash(\tau \wedge \neg(\tau \cap \delta+\psi)) \rightarrow \tau \wedge \neg(\tau \cap \delta)$. Since $\tau \in p^{+}(x)$, and $(\tau \cap \delta)+\psi \in\{\neg \sigma(x) \mid I(U) \vDash \sigma \rightarrow \psi\}$, and $\vDash \tau \wedge \neg(\tau \cap \delta) \rightarrow \neg \delta$, the result follows.

Proposition 8.7. Suppose that $H\left({ }_{R} N\right)=U$ and that $U$ is reflexive. Then ${ }_{R} N \stackrel{f}{\longrightarrow}{ }_{R} M$ is pure iff it is U-pure. 
Proof. Suppose that ${ }_{R} N \stackrel{f}{\longrightarrow}{ }_{R} M$ is $U$-pure, so that $0 \rightarrow D U \otimes N \stackrel{1 \otimes f}{\longrightarrow}$ $D U \otimes M$ is exact. Let $a \in N$ and let $\varphi / \psi \in \operatorname{tp}^{+}(a)$ be a $U$-minimal pair. If we assume that ${ }_{R} N \stackrel{f}{\longrightarrow}{ }_{R} M$ is not pure, then $f$ restricted to $a$ is not a partial isomorphism i.e., $\operatorname{tp}^{+}(a f)$ strictly contains $\operatorname{tp}^{+}(a)$. By Lemma 8.6, we can assume that there is a $\sigma \in \operatorname{tp}^{+}(a f)$ which is not in $\operatorname{tp}^{+}(a)$ and that $I(U) \vDash \sigma \rightarrow \psi$. Replacing $\psi$ with $(\sigma \cap \varphi)+\psi$, we can assume that $M \vDash \psi(a f)$. Let $\Phi(x)=\operatorname{tp}^{+}(a)$ and let $\Psi(x)$ be an ideal of ppfs which is maximal with respect to the properties that $\psi \in \Psi$ and $\Phi \cup\{\neg \sigma \mid \sigma \in \Psi\}$ is $\operatorname{Th}(U)$-consistent. Applying $D$ and Lemma 4.6 at $\operatorname{Th}(U)$, we get an indecomposable type $p(x)$ such that $H(p) \in I(U)^{\prime}, \vDash p^{+}(x) \leftrightarrow D \Psi(x)$ and $p^{-}(x) \supseteq\{D \Phi(x)\}$. But then $H(p) \in(D \psi / D \varphi)$ and $H(p)$ has no choice but to be $D U$. Let $\vDash p(c)$ with $c \in D U$. We know that $D U \vDash D \psi(c)$ so by Proposition 3.2, $c \otimes a f=0$ in $D U_{R} \otimes M$. But, by choice of $\Phi$ and Proposition 3.2, $c \otimes a \neq 0$ in $D U_{R} \otimes N$, contradicting the $U$-purity of ${ }_{R} N \stackrel{f}{\longrightarrow}{ }_{R} M$.

Corollary 8.8. $U$ is $U$-pure-injective.

Proof. $H(U)=U$, so any $U$-pure mapping of $U$ is pure and hence splits, by Proposition 8.7.

In $\S 12$, we treat modules of the form $\operatorname{Hom}(U, E)$, exhibited in Example 8.5. But in the general case, we content ourselves with the next result. There is a natural map $T: D U_{R} \rightarrow \operatorname{Hom}_{S}\left({ }_{R} U_{S}, D U \otimes U_{S}\right)$ which takes $c \in D U$ to the homomorphism $T c$ defined by $T c(a)=c \otimes a$.

Proposition 8.9. If $U$ is reflexive, then $T: D U_{R} \rightarrow \operatorname{Hom}_{S}\left({ }_{R} U_{S}, D U \otimes U_{S}\right)$ is a split imbedding.

Proof. By Corollary 8.8, it is enough to show that $T$ is $D U$-pure, i.e., that $0 \rightarrow D U \otimes U \stackrel{T \otimes 1}{\longrightarrow} \operatorname{Hom}(U, D U \otimes U) \otimes U$ is exact. Let $\mathbf{c} \in D U$ and $\mathbf{a} \in U$ be of the same length and suppose that $T \mathbf{c} \otimes \mathbf{a}=0$ in $\operatorname{Hom}(U, D U \otimes U) \otimes U$. By the definition of tensoring, there is a map $E: \operatorname{Hom}(U, D U \otimes U) \otimes U \rightarrow D U \otimes U$ which is determined by $E(f \otimes c)=f(c)$. Then $\mathbf{c} \otimes \mathbf{a}=E(T \mathbf{c} \otimes \mathbf{a})=0$.

\section{COHERENT RINGS}

In this section, we consider reflection as it pertains to flat and injective modules. In fact, it is the absolutely pure modules that are, in some sense, the reflections of flat modules-a module $M$ is absolutely pure if every imbedding of $M$ is pure.

Proposition 9.1. If $I\left({ }_{R} M\right)^{\prime}$ is a set of flat modules, then $M$ is absolutely pure.

Proof. Let $0 \rightarrow{ }_{R} M \stackrel{f}{\longrightarrow}{ }_{R} N$ be exact. By hypothesis, $0 \rightarrow F \otimes_{R} M \stackrel{1 \otimes f}{\longrightarrow}$ $F \otimes_{R} N$ is exact for each $F \in I\left({ }_{R} M\right)^{\prime}$. Thus $f$ is $I(M)$-pure and hence, by Corollary 8.4, pure.

If $M$ is pure-injective and satisfies the hypothesis of Proposition 9.1, then $M$ is injective. Thus we get 
Proposition 9.2. (i) If $U_{R} \in I_{R}$ is flat and reflexive, then ${ }_{R} D U$ is injective.

(ii) If $I \subseteq I_{R}$ is a closed set of flat modules, then $I^{\prime} \subseteq{ }_{R} I$ is a set of injective modules.

Proof. (ii) is immediate from Proposition 9.1. To prove (i), let $0 \rightarrow{ }_{R} D U \stackrel{f}{\longrightarrow}$ ${ }_{R} M$ be exact. By hypothesis, $0 \rightarrow U \otimes_{R} D U \stackrel{1 \otimes f}{\longrightarrow} U \otimes_{R} M$ is exact and so $f$ is $D U$-pure. But, by Corollary 8.8, $D U$ is $D U$-pure-injective so $f$ splits.

In 1971, Eklof and Sabbagh wrote two articles, [E-S] and its reflection [S-E]. [E-S, Theorem 3.16 (with Proposition 3.23)], states that if $R$ is left coherent, then the class of absolutely pure left $R$-modules is elementary. It follows that the absolutely pure pure-injective i.e., injective, indecomposable left $R$-modules form a closed subset ${ }_{\mathrm{inj}} I$ of ${ }_{R} I$. [S-E, Theorem 4] says that if $R$ is left coherent, then the class of flat right $R$-modules is elementary (in the language $L_{R}$ ). As above, it follows that the flat pure-injective indecomposable right $R$-modules form a closed subset $I_{\text {flat }} \subseteq I_{R}$ of the right Ziegler spectrum. For the purposes at hand, this is all that one needs to know about left coherent rings.

Theorem 9.3. If $R$ is left coherent, then ${ }_{\text {inj }} I^{\prime}=I_{\text {flat }}$. Thus the largest theory $T\left(I_{\text {flat }}\right)$ of flat right $R$-modules is the dual of $T\left(\mathrm{inj}_{\mathrm{inj}} I\right)$, the largest theory of absolutely pure left $R$-modules.

Before proving Theorem 9.3, we make a few observations and recall some facts. For a right module $F_{R}$ and $\varphi \in L_{R}$ in ppf in one variable, denote by $F \varphi(R)$ the subgroup of $F$ consisting of linear combinations of elements in $F$ with coefficients in $\varphi(R)$.

Fact 9.4 [P, Theorem 14.9]. A right module $F$ is flat iff $\varphi(F)=F \varphi(R)$ for each ppf $\varphi \in L_{R}$ in one variable.

If $R$ is left coherent, then by the remarks above, $I\left(R_{R}\right) \subseteq I_{\text {flat }}$. But if $F \in I_{\text {flat }}$ and $F \in(\varphi / \psi)$, then $\varphi(F) \not \subset \psi(F)$ and so $F \varphi(R) \not \subset F \psi(R)$ and hence $\varphi\left(R_{R}\right) \not \subset \psi\left(R_{R}\right)$ i.e., $I\left(R_{R}\right) \cap(\varphi / \psi) \neq \varnothing$. This means that $F$ is in the closure of $I\left(R_{R}\right)$ and hence that $F \in I\left(R_{R}\right)$ and hence that $I_{\text {flat }}=I\left(R_{R}\right)$.

Fact 9.5. If $M$ is injective, $a \in M$, and $p(x)=p p-\operatorname{tp}(a)$, then $p^{+}(M)=$ $\operatorname{ann}_{M}(\operatorname{ann}(a))$.

Proof. $p^{+}(M) \subseteq \operatorname{ann}_{M}(\operatorname{ann}(a))$ is easy to let $\left.c \in \operatorname{ann}_{M}(a)\right)$. By the injectivity of $M$ there is an $f \in \operatorname{End}_{R} M$ such that $a f=c$ and hence $\vDash p^{+}(c)$.

Proof of Theorem 9.3. By Proposition 9.2(ii), $I_{\text {flat }}^{\prime} \subseteq$ inj $I$, so $I_{\text {flat }}=I_{\text {flat }}^{\prime \prime} \subseteq$ inj $I^{\prime}$. To prove the other inclusion, let $F_{R} \in{ }_{\mathrm{inj}} I^{\prime}$ and $\varphi \in L_{R}$ a ppf in one variable. Let $M$ be an $\left|R+\aleph_{0}\right|^{+}$-saturated (and hence pure-injective) model of $T\left({ }_{\mathrm{inj}} I\right)$. Let $a \in M$ be such that $M \vDash \operatorname{tp}^{+}(a) \leftrightarrow D \varphi(x)$. Since $M$ is pure injective and absolutely pure, it is injective and hence $M \vDash \operatorname{tp}^{+}(a) \leftrightarrow \operatorname{ann}(a) x=0$. This follows from Fact 9.5 and the saturation of $M$. Consequently, $M \vDash$ $\operatorname{ann}(a) x=0 \leftrightarrow D \varphi(x)$. Since $D \varphi(x)$ is finitary and $M$ saturated, this means that there are $r_{1}, \ldots, r_{n} \in \operatorname{ann}(a)$ such that $M \vDash D \varphi(x) \leftrightarrow \wedge\left\{r_{i} x=0 \mid 1 \leq i \leq\right.$ $n\}$. Since this equivalence then holds in $T\left({ }_{\text {inj }} I\right)$, an application of $D$ yields ${ }_{\text {inj }} I^{\prime} \vDash \varphi(x) \leftrightarrow \sum\left\{r_{i}|x| 1 \leq i \leq n\right\}$. Because $F_{R} \in$ inj $I^{\prime}$ the same equivalence holds in $\operatorname{Th}(F)$. Therefore $\varphi(F)=\sum F r_{i}$. Similarly, $I_{\text {flat }}=I\left(R_{R}\right)$ implies 
that $R_{R} \vDash \varphi(x) \leftrightarrow \sum\left\{r_{i}|x| 1 \leq i \leq n\right\}$ and hence that $\varphi(R)=\sum R r_{i}$. Thus $\varphi(F)=\sum F r_{i}=\sum F R r_{i}=F\left(\sum R r_{i}\right)=F \varphi(R)$ and $F$ is flat, by Fact 9.4.

Note that in the course of the above proof, we showed that every pp-definable subgroup of $R_{R}, R$ left coherent, is a finitely generated left ideal. The converse holds for arbitrary rings and so we see that $\operatorname{Latt}\left(R_{R}\right)$ is isomorphic to the lattice of finitely generated left ideals of $R$. By Proposition 4.5 and Theorem 9.5, $m-\operatorname{dim}($ inj $I)<\infty$ iff $m$-dim $\left(I_{\text {flat }}\right)<\infty$ iff $m-\operatorname{dim}\left(R_{R}\right)<\infty$ iff the lattice of finitely generated left ideals of $R$ does not contain a dense chain [Z, Lemma 8.5]. Applying the considerations made at the end of $\S 4$ gives us the following

Corollary 9.6. If $R$ is left coherent and the lattice of finitely generated left ideals does not contain a dense chain, then duality constitutes a bijection between the (isomorphism types of) injective indecomposable left $R$-modules and the (isomorphism types of ) flat pure-injective indecomposable right $R$-modules.

Corollary 9.6 applies, in particular, to left noetherian rings.

\section{TOTALLY TRANSCENDENTAL MODULES}

In this section, we specialize to the case of totally transcendental modules. Recall [P, Theorem 3.1] that a module is totally transcendental iff it has the descending chain condition on pp-definable subgroups. Every totally transcendental module $M$ is pure-injective and $m-\operatorname{dim}(M)<\infty$ so that every $U \in I(M)$ is reflexive. A main feature of a totally transcendental module $M$ is that for each $\mathbf{a} \in M$, there is a ppf $\varphi(\mathbf{x})$ such that $M \vDash \varphi(\mathbf{x}) \leftrightarrow \operatorname{tp}^{+}(\mathbf{a})$. We shall regularly exploit the fact [Z, Corollary 3.3] that $c \in \varphi(M)$ iff there is an $f \in \operatorname{End}_{R} M$ such that $\mathbf{a} f=\mathbf{c}$. We begin with a closer look at the situation in Proposition 8.9 by showing that in this case, elements of $\operatorname{Hom}_{S}\left({ }_{R} U_{S}, D U \otimes U_{S}\right)$ locally resemble elements of $D U$. Throughout this section and the next, $S$ will denote the endomorphism ring of ${ }_{R} U$.

Lemma 10.1. Suppose that ${ }_{R} U$ is a totally transcendental indecomposable module and that $f \in \operatorname{Hom}_{S}\left(U_{S}, D U \otimes U_{S}\right)$. Then for each $a \in U$, there is a $c \in D U$ such that $f(a)=c \otimes a$.

Proof. Let $f(a)=\mathbf{b} \otimes \mathbf{e}$ and let $\rho(\mathbf{x}, y)$ be a ppf such that $U \vDash \rho(\mathbf{x}, y) \leftrightarrow$ $\operatorname{tp}^{+}(\mathbf{e}, a)$. We aim to show that $D(\rho(\mathbf{x}, 0)) \in \operatorname{tp}^{+}(\mathbf{b})$. By Theorem 4.10, it suffices to prove that for each $\mathbf{d} \in U$ for which $U \vDash \rho(\mathbf{d}, 0)$, we have that $\mathbf{b} \otimes \mathbf{d}=0$ in $D U \otimes U$. But if $U \vDash \rho(\mathbf{d}, 0)$, then by the choice of $\rho(\mathbf{x}, y)$, there is a $g \in \operatorname{End}_{R} U$ such that $\mathbf{e} g=\mathbf{d}$ and $a g=0$ and so $\mathbf{b} \otimes \mathbf{d}=\mathbf{b} \otimes \mathbf{e} g=$ $f(a g)=0$. By Corollary 2.8(ii) this means that $D U \vDash \exists y D \rho(\mathbf{b}, y)$ and if $c \in D U$ witnesses this, then Corollary 3.3 gives that $f(a)=\mathbf{b} \otimes \mathbf{e}=(c) D \rho \otimes \mathbf{e}=$ $c \otimes \rho \mathbf{e}=c \otimes a$.

If $U$ is totally transcendental, we may define elementary socles on it.

Definition 10.2. For each ordinal $\alpha$, we define $\operatorname{soc}^{\alpha}(U) \subseteq U$ by recursion on $\alpha$.

(i) $\operatorname{soc}^{0}(U)=0$.

(ii) $\operatorname{soc}^{\alpha+1}(U)=\sum\left\{\varphi(U) \mid \varphi(U) \not \subset \operatorname{soc}^{\alpha}(U)\right.$ and $\varphi(U)$ is minimal such $\}+$ $\operatorname{soc}^{\alpha}(U)$.

(iii) $\operatorname{soc}^{\lambda}(U)=\sum\left\{\operatorname{soc}^{\alpha}(U) \mid \alpha<\lambda\right\}$ if $\lambda$ is a limit ordinal. 
$\operatorname{ht}(U)$ is defined as the least $\alpha$ for which $\operatorname{soc}^{\alpha}(U)=U$ and $\operatorname{rk}(\varphi(U))$ will denote the foundation rank of $\varphi(U)$ in $\operatorname{Latt}(U)$. Since everything is defined inside $U$, one may prove by induction on $\alpha$ that if $\operatorname{rk}(\varphi(U)) \leq \alpha$, then $\varphi(U) \subseteq \operatorname{soc}^{\alpha}(U)$. We shall often confuse $\operatorname{soc}^{\alpha}(U)$ with the ideal $\{\psi \mid \psi(U) \subseteq$ $\left.\operatorname{soc}^{\alpha}(U)\right\}$. Since $\operatorname{Latt}(D U) \cong \operatorname{Latt}(U)^{\text {op }}$ (Theorem 4.10), we may dually define the elementary radicals $\operatorname{rad}^{\alpha}(D U)$ of $D U$.

Definition 10.3. $\operatorname{rad}^{\alpha}(D U)=D\left(\operatorname{soc}^{\alpha}(U)\right)(D U)$, the subgroup of $D U$ defined by the filter of ppfs dual to the ideal $\operatorname{soc}^{\alpha}(U)$. We would like to be able to confuse $\operatorname{rad}^{\alpha}(D U)$ with the filters $D\left(\operatorname{soc}^{\alpha}(U)\right)$ and $\left\{D \varphi \mid D \varphi(D U) \supseteq \operatorname{rad}^{\alpha}(D U)\right\}$ and we may, according to

Proposition 10.4. $\varphi \in \operatorname{soc}^{\alpha}(U)$ iff $D \varphi(D U) \supseteq \operatorname{rad}^{\alpha}(D U)$.

Proof. $(\Rightarrow)$ follows from the definition so we just prove $(\Leftarrow)$. Suppose that $\varphi \notin \operatorname{soc}^{\alpha}(U)$ is a minimal such $\varphi$. Let $a \in \varphi(U) \backslash \operatorname{soc}^{\alpha}(U)$. Then $U \vDash$ $\varphi \leftrightarrow \operatorname{tp}^{+}(a)$ and we can find $\psi$ such that $\varphi / \psi \in \operatorname{tp}(a)$ is a $U$-minimal pair. By the minimality of $\varphi, \psi \in \operatorname{soc}^{\alpha}(U)$. Lemma 4.6 at $\operatorname{Th}(U)$ provides us with an indecomposable type $p, H(p) \in I(U)^{\prime}=I(D U)$, for which $\vDash p^{+} \rightarrow$ $D\left(\operatorname{soc}^{\alpha}(U)\right)$ and $\neg D \varphi \in p$. As $D \psi / D \varphi \in p$ it must be that $H(p)=D U$ and if $c \in D U$ is a realization of $p$, then $c \in \operatorname{rad}^{\alpha}(D U)$, but $c \notin D \varphi(D U)$.

It is sometimes easier to determine $D U \otimes U$ than $D U$.

Theorem 10.5. If $U$ is a totally transcendental indecomposable module, then $D U_{R} \cong \operatorname{Hom}_{S}\left({ }_{R} U_{S}, D U \otimes U_{S}\right)$.

Proof. Let $f \in \operatorname{Hom}(U, D U \otimes U)$. For each ordinal $\alpha$, we shall provide an element $c_{\alpha} \in D U$ such that $\left(f-T c_{\alpha}\right)\left(\operatorname{soc}^{\alpha}(U)\right)=0 \quad(T$ is defined prior to Proposition 8.9). Letting $\alpha=\operatorname{ht}(U)$ together with Proposition 8.9 will yield the theorem. We proceed by induction on $\alpha$.

(i) $c_{0}=0$.

(ii) For the successor case, it suffices to prove that $f\left(\operatorname{soc}^{\alpha}(U)\right)=0$ implies that there is a $c \in D U$ such that $(f-T c)\left(\operatorname{soc}^{\alpha+1}(U)\right)=0$. Then, if we replace $f$ with $f-T c_{\alpha}$ and let $c_{\alpha+1}=c_{\alpha}+c$, the induction goes through.

Let $F=\left\{\varphi_{\gamma}(U) \mid \gamma<\kappa, \varphi_{\gamma}(U) \not \subset \operatorname{soc}^{\alpha}(U)\right.$ is minimal such $\}$ be a family of pp-definable subgroups of $U$ maximal with respect to the property that for each finite $J \subseteq \kappa$ and $\gamma_{0} \notin J, \varphi_{\gamma_{0}}(U) \not \subset \sum\left\{\varphi_{\gamma}(U) \mid \gamma \in J\right\}+\operatorname{soc}^{\alpha}(U)$. By the maximality of $F$, $\operatorname{soc}^{\alpha}(U)+F=\operatorname{soc}^{\alpha+1}(U)$. Let $\gamma_{0}<\kappa$ and $a_{\gamma_{0}} \in$ $\varphi_{\gamma_{0}}(U) \backslash \operatorname{soc}^{\alpha}(U)$. By the minimality of $\varphi_{\gamma_{0}}(U), U \vDash \varphi_{\gamma_{0}} \leftrightarrow \operatorname{tp}^{+}\left(a_{\gamma_{0}}\right)$ and if $\varphi_{\gamma_{0}} / \psi_{\gamma_{0}} \in \operatorname{tp}\left(a_{\gamma_{0}}\right)$ is a $U$-minimal pair, then $\psi_{\gamma_{0}}(U) \subseteq \operatorname{soc}^{\alpha}(U)$.

By Lemma 10.1, there is an $e_{\gamma_{0}} \in D U$ such that $f\left(a_{\gamma_{0}}\right)=e_{\gamma_{0}} \otimes a_{\gamma_{0}}$. As each $m \in \psi_{\gamma_{0}}(U)$ may written as $a_{\gamma_{0}} g$ with $g \in S=\operatorname{End}_{R} U$, we get that $e_{\gamma_{0}} \otimes m=e_{\gamma_{0}} \otimes a_{\gamma_{0}} g=f\left(a_{\gamma_{0}}\right) g=f\left(a_{\gamma_{0}} g\right)=f(m)=0$, by assumption, so that Theorem 4.10 implies that $e_{\gamma_{0}} \in D \psi_{\gamma_{0}}(D U)$ and Proposition 3.2 gives that if $f\left(a_{\gamma_{0}}\right) \neq 0$, then $D \psi_{\gamma_{0}} / D \varphi_{\gamma_{0}} \in \operatorname{tp}\left(e_{\gamma_{0}}\right)$. If $\psi \in \operatorname{rad}^{\alpha}(D U)$ is such that $\vDash \psi \rightarrow D \psi_{\gamma_{0}}$ and $J \subseteq \kappa$ is finite, then, by the definition of $F$, it follows that $\varphi_{\gamma_{0}}(U) \not \subset \sum\left\{\varphi_{\gamma}(U) \mid \gamma \in J\right\}+D \psi(U)$ so that $\bigcap\left\{D \varphi_{\gamma}(D U) \mid \gamma \in J\right\} \cap \psi(D U) \not \subset$ $D \varphi_{\gamma_{0}}(D U)$, by Theorem 4.10. Now the same argument used in part (a) of the proof of Proposition 6.4 shows that $\left\{e_{\gamma_{0}}=x+y, D \varphi_{\gamma_{0}}(y), x \in \operatorname{rad}^{\alpha}(D U)\right.$, $\left.D \varphi_{\gamma}(x) \mid \gamma \neq \gamma_{0}\right\}$ is finitely satisfied in $D U$ and hence, by Proposition 0.4, realized by say $\left(e_{\gamma_{0}}^{\prime}, e_{\gamma_{0}}-e_{\gamma_{0}}^{\prime}\right) \in D U$. Then we have that $e_{\gamma_{0}}^{\prime} \in \operatorname{rad}^{\alpha}(D U)$, 
$D U \vDash D \varphi_{\gamma}\left(e_{\gamma_{0}}^{\prime}\right)$ for each $\gamma \neq \gamma_{0}$ and $D \varphi_{\gamma_{0}}\left(e_{\gamma_{0}}-e_{\gamma_{0}}^{\prime}\right)$ so that, by Proposition 3.2, $f\left(a_{\gamma_{0}}\right)=e_{\gamma_{0}} \otimes a_{\gamma_{0}}=e_{\gamma_{0}}^{\prime} \otimes a_{\gamma_{0}}$. If $f\left(a_{\gamma_{0}}\right)=0$, let $e_{\gamma_{0}}^{\prime}=0$.

Set $e_{\gamma_{0}}=e_{\gamma_{0}}^{\prime}$. Thus we may assume that we have for each $\gamma<\kappa, e_{\gamma} \in D U$ such that $f\left(a_{\gamma}\right)=e_{\gamma} \otimes a_{\gamma}, e_{\gamma} \in \operatorname{rad}^{\alpha}(D U)$ and that $D U \vDash D \varphi_{\delta}\left(e_{\gamma}\right)$ for each $\delta \neq \gamma$. It is easy to see now that $\left\{x \in \operatorname{rad}^{\alpha}(D U)\right\} \cup\left\{D \varphi_{\gamma}\left(x-e_{\gamma}\right) \mid \gamma<\kappa\right\}$ is finitely satisfied in $D U$. In fact, if $J \subseteq \kappa$ is finite, then $\sum\left\{e_{\gamma} \mid \gamma \in J\right\}$ is a realization of $\left\{x \in \operatorname{rad}^{\alpha}(D U)\right\} \cup\left\{D \varphi_{\gamma}\left(x-e_{\gamma}\right) \mid \gamma \in J\right\}$. By Proposition 0.4, there is a $c \in D U$ such that $c \in \operatorname{rad}^{\alpha}(D U)$ and $D U \vDash D \varphi_{\gamma}\left(c-e_{\gamma}\right)$ for each $\gamma<\kappa$. Since each $m \in \varphi_{\gamma}(U)$ may written as $a_{\gamma} g$ with $g \in S=\operatorname{End}_{R} U$, it follows that $\operatorname{soc}^{\alpha+1}(U)=\sum\left\{a_{y} S \mid \gamma<\kappa\right\}+\operatorname{soc}^{\alpha}(U)$. So for each $b \in \operatorname{soc}^{\alpha+1}(U)$, there is a finite $J \subseteq \kappa$ and $a \in \operatorname{soc}^{\alpha}(U)$ such that $b=\sum\left\{a_{\gamma} g_{\gamma} \mid \gamma \in J\right\}+a$ with $g_{\gamma} \in S$ and therefore $f(b)=f\left(\sum\left\{a_{\gamma} g_{\gamma} \mid \gamma \in J\right\}+a\right)=\sum\left\{f\left(a_{\gamma}\right) g_{\gamma} \mid \gamma \in\right.$ $J\}+f(a)=\sum\left\{e_{\gamma} \otimes g_{\gamma} \mid \gamma \in J\right\}+0=\sum\left\{c \otimes g_{\gamma} \mid \gamma \in J\right\}+c \otimes a$, by choice of $c$ and Proposition 3.2. Thus $f(b)=c \otimes b$ and hence $(f-T c)\left(\operatorname{soc}^{\alpha+1}(U)\right)=0$.

(iii) Suppose that $\lambda$ is a limit ordinal and that for each $\alpha<\lambda$, we are given an element $c_{\alpha} \in D U$ such that $\left(f-T c_{\alpha}\right)\left(\operatorname{soc}^{\alpha}(U)\right)=0$. We claim that $\left\{x-c_{\alpha} \in \operatorname{rad}^{\alpha}(D U) \mid \alpha<\lambda\right\}$ is finitely satisfied in $D U$. This is so because if $\gamma<\delta<\lambda$, then $T\left(c_{\gamma}-c_{\delta}\right)\left(\operatorname{soc}^{\gamma}(U)\right)=0$ i.e., for each $\varphi \in \operatorname{soc}^{\gamma}(U)$, $\left(c_{\gamma}-c_{\delta}\right) \otimes \varphi(U)=0$ in $D U \otimes U$. By Theorem 4.10, this means that $\left(c_{\gamma}-c_{\delta}\right) \in$ $D \varphi(D U)$ for every such $\varphi$ and hence that $\left(c_{\gamma}-c_{\delta}\right) \in \operatorname{rad}^{\alpha}(D U)$. Thus if $\alpha_{1}<\alpha_{2}<\cdots<\alpha_{n}=\delta<\lambda$ is a finite sequence of ordinals less than $\lambda$, then $c_{\delta}$ realizes $\left\{x-c_{\alpha_{i}} \in \operatorname{rad}^{\alpha_{i}}(D U) \mid 1 \leq i \leq n\right\}$. Let $c_{\lambda} \in D U$ realize $\left\{x-c_{\alpha} \in\right.$ $\left.\operatorname{rad}^{\alpha}(D U) \mid \alpha<\lambda\right\}$. Then for each $\alpha<\lambda, T\left(c_{\lambda}-c_{\alpha}\right)\left(\operatorname{soc}^{\alpha}(U)\right)=0$ and hence for each $\alpha<\lambda,\left(f-T c_{\lambda}\right)\left(\operatorname{soc}^{\alpha}(U)\right)=0$ and hence $\left(f-T c_{\lambda}\right)\left(\operatorname{soc}^{\lambda}(U)\right)=0$.

If $\varphi / \psi$ is a ${ }_{R} U$-minimal pair, then we see from the proof of Proposition 6.4 how $\varphi(U) / \psi(U)$ is a one-dimensional right vector space over $\Delta$, the residue division ring of $S=\operatorname{End}_{R} U$, and how $D \psi(D U) / D \varphi(D U)$ is a one-dimensional left vector space over $\Delta$. Thus $D \psi(D U) \otimes \varphi(U)$ is a (onedimensional) $\Delta$ - $\Delta$-bimodule when viewed as a submodule of the $S^{\prime}-S$-bimodule $D U \otimes U$ where $S^{\prime}=\operatorname{End}_{R} D U$. We shall refer to $D \psi(D U) \otimes \varphi(U)$ as $S_{S^{\prime}} \Delta_{S}$. This does not, however, give $\varphi / \psi$ any prominence among $U$-minimal pairs, for if $\sigma / \tau$ is another such pair, then [Z, Corollary 4.6] supplies us with a third $U$-minimal pp-pair $\delta / \varepsilon$ such that $\delta / \varepsilon \leq_{I} \varphi / \psi$ and $\delta / \varepsilon \leq_{I} \sigma / \tau$ (Definition 3.5 localized at $I=I(U))$. By Proposition 3.6(ii) at $I, D \varepsilon(D U) \otimes \delta(U)$ is an $S^{\prime}$-S -submodule of both $D \psi(D U) \otimes \varphi(U)$ and $D \tau(D U) \otimes \sigma(U)$ and hence $D \tau(D U) \otimes \sigma(U)={ }_{S} \Delta_{S}$.

In the following, $E(M)$ denotes the injective hull of $M$.

Proposition 10.6. If $U$ is a totally transcendental indecomposable module, then $E\left(D U \otimes U_{S}\right)=E\left(\Delta_{S}\right)$ as right $S$-modules.

Proof. We will show that for each $\mathbf{a} \otimes \mathbf{b} \in D U \otimes U$, there is an $f \in S$ such that $\mathbf{a} \otimes \mathbf{b} f \in \Delta_{S}$, i.e., that $D U \otimes U_{S}$ is an essential extension of $\Delta_{S}$. Suppose that $U \vDash \varphi / \psi \leftrightarrow \operatorname{pp}-\operatorname{tp}(\mathbf{b})$ and $U \vDash \varphi \leftrightarrow \operatorname{tp}^{+}(\mathbf{b})$. If $\mathbf{a} \otimes \mathbf{b} \neq 0$ in $D U \otimes U$, then $D U \vDash \neg D \varphi(\mathbf{a})$, by Proposition 3.2. By [Z, Corollary 4.6], there is a $D U$ minimal pair $D \tau / D \sigma \in \operatorname{tp}(\mathbf{a})$ such that $D \varphi \subseteq D \sigma \subseteq D \tau$. Then $\tau \subseteq \sigma \subseteq \varphi$ and $\sigma / \tau$ is $U$-minimal. Let $\mathbf{c} \in \sigma(U) \backslash \tau(U)$. Then $U \vDash \varphi(\mathbf{c})$ so there is an $f \in S$ such that $\mathbf{b} f=\mathbf{c}$ and hence it follows that $\mathbf{a} \otimes \mathbf{b} f=\mathbf{a} \otimes \mathbf{c} \in D \tau(D U) \otimes \sigma(U)=$ $\Delta_{S}$. 
If $U$ is an indecomposable injective left $R$-module over a left noetherian ring $R$, then, according to Fact 9.5 , all of its pp-definable subgroups have the form $J x=0, J$ a left ideal of $R$. Thus $U$ is totally transcendental and the above results apply.

\section{Finitely GENERATED MOdUlES}

We now solve Problem 4.11 for a certain class of modules. $S=\operatorname{End}_{R} U$ in this section.

Definition 11.1. A module $M$ is definably finitely generated by a $\in M$ if for each $c \in M$, there is a ppf $\rho(\mathbf{a}, x) \in \operatorname{tp}^{+}(c / \mathbf{a})$ and $M \vDash \rho(0, x) \leftrightarrow x=0$.

If $M$ is definably finitely generated by $\mathbf{a}$, then $M$ is the positive definable closure of a. If $M$ is finitely generated as an $R$-module by $\mathbf{a}$, then it is definably finitely generated by $\mathbf{a}$. If $M$ is definably finitely generated, then $|M| \leq|R|+\aleph_{0}$, the cardinality of the language ${ }_{R} L$, so if $R$ is countable and $U$ is a pure-injective indecomposable which is definably finitely generated, then $U$ must be totally transcendental. This does not hold for uncountable $R$ and not every totally transcendental module is definably finitely generated $\left(Z\left(p^{\infty}\right)\right.$, for example).

Theorem 11.2. If ${ }_{R} U_{S}$ is a definably finitely generated totally transcendental indecomposable module, then $D U \otimes U_{S}=E\left(\Delta_{S}\right)$.

Proof. Let $U$ be definably generated by a. Then $D U \otimes U=D U \otimes \mathbf{a}$, for if $e \otimes c \in D U \otimes U$ is an elementary tensor, then there is a ppf $\rho(\mathbf{a}, x) \in \operatorname{tp}^{+}(c / \mathbf{a})$ such that $U \vDash \rho(0, x) \leftrightarrow x=0$. By Theorem 4.10 and Corollary $2.8 D U \vDash$ $\exists \mathbf{y} D \rho(\mathbf{y}, x) \leftrightarrow x=x$ and so $e \otimes c=e \otimes \rho(\mathbf{a})=e D \rho \otimes \mathbf{a}$. Choose $\varphi(\mathbf{x})$ such that $U \vDash \varphi(\mathbf{x}) \leftrightarrow \operatorname{tp}^{+}(\mathbf{a})$. We have proved that $D U \otimes U=(D U)^{n} \otimes \varphi(U)=$ $\left((x=x)^{n} \otimes \varphi\right)(D U \times U)$ (in the sense of $\left.\S 3\right)$ where $n=l(\mathbf{a})$.

Next we see that $\varphi(U)_{S} \cong S_{S}$. This is so because for each $c \in \varphi(U)$ there is, by choice of $\varphi$, an $f \in S$ for which $\mathbf{a} f=\mathbf{c}$. If $\mathbf{c}=0$ and $e \in U$ is arbitrary, then considering a ppf $\rho(\mathbf{a}, x) \in \operatorname{tp}^{+}(e / \mathbf{a})$ such that $U \vDash \rho(0, x) \leftrightarrow x=0$ shows that $U \vDash \rho(\mathbf{a} f, e f)$ and hence that $e f=0$ and that $f=0$.

It follows that $R(\varphi)^{I} \subseteq \operatorname{End}_{S} \varphi(U)_{S}$ where $I=I(U)$ and $\varphi$ is an abbreviation for $\varphi /(x=0)^{n}$. We claim that, in fact, equality holds, for if $c \in \varphi(U)$ and $\rho(\mathbf{a}, \mathbf{y}) \in \operatorname{tp}^{+}(\mathbf{c} / \mathbf{a})$ is such that $U \vDash \rho(0, \mathbf{y}) \leftrightarrow \mathbf{y}=0$ then the following four conditions are satisfied.

(i) $I \vDash \varphi(\mathbf{x}) \rightarrow \exists \mathbf{y} \rho(\mathbf{x}, \mathbf{y})$,

(ii) $I \vDash \mathbf{x}=0 \rightarrow \rho(\mathbf{x}, 0)$,

(iii) $I \vDash \exists \mathbf{x} \rho(\mathbf{x}, \mathbf{y}) \rightarrow \varphi(\mathbf{y})$,

(iv) $I \vDash \rho(0, \mathbf{y}) \rightarrow \mathbf{y}=0$.

Thus $\rho(\mathbf{x}, \mathbf{y})$ represents an element $\rho \in R(\varphi)^{I}$ for which $\rho(\mathbf{a})=\mathbf{c}$.

Thus we have that $R(\varphi)^{I} \cong S$ and ${ }_{S} \varphi(U)_{S} \cong S$. It was noted earlier (just after Proposition 3.4) how $\eta:(D U)^{n} / D \varphi(D U)_{S} \cong(D U)^{n} / D \varphi(D U)_{S} \otimes S \rightarrow$ $(D U)^{n} / D \varphi(D U) \otimes \varphi(U)=D U \otimes U_{S}$ induced by $\eta(\mathbf{e})=\mathbf{e} \otimes \mathbf{a}$ is surjective. On the other hand if $\eta(\mathbf{e})=\mathbf{e} \otimes \mathbf{a}=0$, then Proposition 3.2 forces $D U \vDash D \varphi(\mathbf{e})$ so that $\eta$ is injective as well. By Proposition 6.7, $\operatorname{Th}\left((D U)^{n} / D \varphi(D U)_{S}\right)=$ $D \operatorname{Th}\left({ }_{s} \varphi(U)\right)=D \operatorname{Th}\left({ }_{s} S\right)=\operatorname{Th}\left(D\left({ }_{S} S\right)\right)$. But by the isomorphism and Proposition 10.6, $(D U)^{n} / D \varphi(D U)_{S}$ is a pure-injective indecomposable right $S$-module. It is pure injective because, by Lemma 6.8, every $S$-pp-type $p^{+}(x / A), A \subseteq$ 
$(D U)^{n} / D \varphi(D U)_{S}$ is induced by a pp-type $\operatorname{tp}^{+}(\mathbf{e} / C), C \subseteq(D U)^{n}, \mathbf{e} \in(D U)^{n}$ and hence realized by $\mathrm{e}+D \varphi(D U)_{S}$. It must be that $(D U)^{n} / D \varphi(D U)_{S}=$ $D\left({ }_{s} S\right)$. By Proposition 9.2(i), $(D U)^{n} / D \varphi(D U)_{S}$ is injective. The theorem now follows from Proposition 10.6.

In the proof of the previous theorem, we used without mentioning the fact that ${ }_{S} S$ is a reflexive pure-injective module. This is obvious just as soon as we extract the following consequence of the proof of Theorem 11.2.

Corollary 11.3. If ${ }_{R} U_{S}$ a totally transcendental indecomposable which is definably finitely generated by $\mathbf{a}, l(\mathbf{a})=n$, then ${ }_{S} S$ is totally transcendental as well and $\mathrm{rk}_{S} S \leq n \cdot \operatorname{rk}(U)$.

Proof. We interpreted the module ${ }_{S} S$ as the left $R(\varphi)^{I}$-module $\varphi(U)$. By Lemma 6.8, each pp-definable subgroup of ${ }_{S} S$ is defined on $\varphi(U)$ by a ppf in ${ }_{R} L$. If $l(\mathbf{a})=n$, then $\operatorname{rk}\left({ }_{S} S\right) \leq \operatorname{rk}(\varphi(U)) \leq n \cdot \operatorname{rk}(U)$.

A ring $R$ is said to be left pure semisimple if every left $R$-module is a direct sum of indecomposable modules. In this case, every $U \in{ }_{R} I$ is totally transcendental and finitely generated $[\mathrm{P}, \S 11.1]$. In fact $m-\operatorname{dim}\left({ }_{R} I\right)<\infty$ so that all left and right pure-injective indecomposables are reflexive.

Corollary 11.4. Let $R$ be a left pure semisimple ring. Then the duality $D$, which takes the indecomposable left $R$-module $U$ to $D U_{R} \cong \operatorname{Hom}_{S}\left({ }_{R} U_{S}, E(\Delta)_{S}\right)$, is a homeomorphism between ${ }_{R} I$, the left Ziegler spectrum and $I_{R}$, the right Ziegler spectrum.

Example 11.5. If $U$ is definably finitely generated and $m$-dim $(U)=0$, then $m-\operatorname{dim}(D U)=0$, but $D U$ need not be definably finitely generated. The following is an example where $U$ is only finitely generated. This is a counterexample to the mistake [H, Proposition 1.11.1].

Let $K / L$ be an infinite dimensional field extension of $L$ with $L \cong K$. Let $i: L \rightarrow K$ be an isomorphism. Let the ring be $R=\left\{\left(\begin{array}{cc}i(n) & k \\ 0 & n\end{array}\right) \mid k \in K, n \in L\right\}$. $R_{R}$ will play the role of the $U$ above. Note that $R$ is a local ring with maximal ideal $J(R)=\left(\begin{array}{ll}0 & K \\ 0 & 0\end{array}\right)$ satisfying $J(R)^{2}=0$ and $R / J(R) \cong{ }_{L} L_{L}$. Now ${ }_{R} J(R) \cong$ ${ }_{L} K$ where the left action of $L$ on $K$ is got from ${ }_{K} K$ by restriction of scalars along $i$. Thus ${ }_{R} J(R)$ is simple and ${ }_{R} R$ is artinian with a composition series of length 2 . On the other hand, $J(R)_{R} \cong K_{L}$ where the right action of $L$ on $K$ is that induced by field multiplication. Thus $J(R)_{R}$ is not finitely generated and $R_{R}$ is not artinian.

The pp-lattice of $R_{R}$ looks like the lattice of left ideals of $R$. It consists of three points $x=0 \subseteq \varphi(x) \subseteq x=x$ where $R_{R} \vDash \varphi(x) \leftrightarrow r \mid x$ for every $r \in$ $J(R), r \neq 0$. By Proposition 9.2(i), ${ }_{R} E=D\left(R_{R}\right)$ is injective and its pp-lattice consists of three points $x=0 \subseteq \psi(x) \subseteq x=x$ where ${ }_{R} E \vDash \psi(x) \leftrightarrow r x=0$ for every $r \in J(R), r \neq 0$.

${ }_{R} M={ }_{R} J(R)$ is the unique (up to isomorphism) simple left $R$-module. Since every left $R$-module contains a copy of $M, E=E(M)$ where $E(M)$ denotes the injective hull of $M$. Let $N=\left\{\left(\begin{array}{l}k_{1} \\ k_{2}\end{array}\right) \mid k_{i} \in K\right\}$ and define the action of $R$ on $N$ by

$$
\left(\begin{array}{cc}
i(n) & k \\
0 & n
\end{array}\right)\left(\begin{array}{l}
k_{1} \\
k_{2}
\end{array}\right)=\left(\begin{array}{c}
i(n) k_{1}+k_{2} \\
n k_{2}
\end{array}\right) .
$$


Consider $\left(\begin{array}{ll}K & 0\end{array}\right)=\left\{\left(\begin{array}{l}k \\ 0\end{array}\right) \mid k \in K\right\} \subseteq N$. It is a simple submodule which is ppdefinable- $(K 0)=\psi(N)$ for any $\psi(x)$ of the form $r x=0, r \in J(R), r \neq 0$. $N$ is an essential extension of $(K 0) \cong M$ so that $E(N)=E(M)=E$. If we think of $N$ as a submodule of $E$, then $(K 0)$ coincides with $M$. We claim that $N=E$. For, let $c \in E$. There is an $r \in R$ such that $r c \in M, r c \neq 0$. If $r$ is a unit, then $c \in M \subseteq N$. Otherwise, $r=\left(\begin{array}{ll}0 & k \\ 0 & 0\end{array}\right) \in J(R)$ and $r c=\left(\begin{array}{c}k^{\prime} \\ 0\end{array}\right)$, say. But then $r\left(\begin{array}{c}0 \\ k^{-1} k^{\prime}\end{array}\right)=r c$ so $r\left(c-\left(\begin{array}{c}k^{\prime} \\ 0\end{array}\right)\right)=0$ and $c-\left(\begin{array}{c}k^{\prime} \\ 0\end{array}\right) \in M$ and hence $c \in N$. Thus $N=E$.

By looking at the bottom component of elements in $E$, it it clear that $E$ is not finitely generated. Indeed, it is not even definably finitely generated. For, if $c, \mathbf{e} \in E$ and $c \notin R \mathbf{e}=\Sigma R e_{i}$, then $R c+R \mathbf{e} / R \mathbf{e}$ is isomorphic to $M$ whence arises a homomorphism $f$ from $R c+R \mathbf{e}$ to $M$ which kills e but $f(c) \neq 0$. $f$ lifts to an endomorphism of $E$. Thus $c$ cannot be pp-definable from $\mathbf{e}$.

\section{MoRItA DUALITY}

Recent work of Zimmermann-Huisgen and Zimmermann deals with the pplattice of the right $R$-module $\operatorname{Hom}_{S}\left({ }_{R} M_{S}, E_{S}\right)$ when $E_{S}$ is injective; $S$ is a ring making ${ }_{R} M_{S}$ into a bimodule.

Proposition 12.1 [ZH-Z, Lemma 2]. If $E_{S}$ is injective, then $D \varphi\left(\operatorname{Hom}_{S}\left({ }_{R} M_{S}, E_{S}\right)\right)$ $=\operatorname{Hom}_{S}\left(M / \varphi(M), E_{S}\right)$. Thus $I\left(\operatorname{Hom}_{S}\left({ }_{R} M_{S}, E_{S}\right)\right) \subseteq I(M)^{\prime}$ and if $E_{S}$ is a cogenerator, equality holds.

Proof. $D \varphi\left(\operatorname{Hom}_{S}\left({ }_{R} M_{S}, E_{S}\right)\right)=\operatorname{Hom}_{S}\left(M / \varphi(M), E_{S}\right)$ follows from the proof of $\left[\mathrm{ZH}-\mathrm{Z}\right.$, Lemma 2]. Since $E_{S}$ is injective, $(\varphi / \psi) \cap I(M)=\varnothing$ iff $\varphi(M) \subseteq$ $\psi(M)$ implies that

$$
\operatorname{Hom}_{S}\left(M / \psi(M), E_{S}\right) \subseteq \operatorname{Hom}_{S}\left(M / \varphi(M), E_{S}\right)
$$

iff

$$
D \psi\left(\operatorname{Hom}_{S}\left({ }_{R} M_{S}, E_{S}\right)\right) \subseteq D \varphi\left(\operatorname{Hom}_{S}\left({ }_{R} M_{S}, E_{S}\right)\right)
$$

iff

$$
(D \psi / D \varphi) \cap I\left(\operatorname{Hom}_{S}\left({ }_{R} M_{S}, E_{S}\right)\right)=\varnothing,
$$

thus

$$
I\left(\operatorname{Hom}_{S}\left({ }_{R} M_{S}, E_{S}\right)\right) \subseteq I(M)^{\prime} .
$$

If, in addition, $E_{S}$ is a cogenerator, then all the above implications become equivalences and we get that $I\left(\operatorname{Hom}_{S}\left({ }_{R} M_{S}, E_{S}\right)\right)=I(M)^{\prime}$.

Corollary 12.2. If $M_{R}$ is injective, then $I(M)^{\prime} \subseteq I\left({ }_{R} R\right)$. If $M$ is a cogenerator, equality holds.

Proof. Apply Proposition 12.1 to $M_{R} \cong \operatorname{Hom}\left({ }_{R} R_{R}, M_{R}\right)$.

If $S$ and $T$ are arbitrary rings, recall that the functor $\operatorname{Hom}_{S}\left(-,{ }_{T} C_{S}\right)$ is equivalent to a Morita duality between $T$-mod and $\bmod -S$ iff ${ }_{T} C_{S}$ is an injective cogenerator as both $T$-module and $S$-module and $T \cong$ End $_{S} C_{S}$ and $S \cong \operatorname{End}_{T} C[\mathrm{~F}, 23.16]$. The functor $\operatorname{Hom}_{S}\left(-,{ }_{T} C_{S}\right)$ is called a ${ }_{T} C_{S}$-duality. 
Proposition 12.3. Suppose that ${ }_{T} C_{S}$-duality constitutes a Morita duality between $T$-mod and mod-S. If ${ }_{R} U_{S}$ is a reflexive pure-injective indecomposable $R$ module which is ${ }_{T} C_{S}$-reflexive, then ${ }_{T} D U_{R} \cong \operatorname{Hom}_{S}\left({ }_{R} U_{S},{ }_{T} C_{S}\right)$ and ${ }_{R} U_{S} \cong$ $\operatorname{Hom}_{T}\left({ }_{T} D U_{R},{ }_{T} C_{S}\right)$.

Proof. As in Example 8.5, $\operatorname{Hom}_{S}\left({ }_{R} U_{S},{ }_{T} C_{S}\right)$ is pure-injective. From the hypothesis and Proposition 12.1, I( $\left.\operatorname{Hom}_{S}\left({ }_{R} U_{S},{ }_{T} C_{S}\right)\right)=I(U)^{\prime}$. By [F, 23.15], the class of ${ }_{T} C_{S}$-reflexive $T$-modules is a Serre class forcing $\operatorname{Hom}_{S}\left({ }_{R} U_{S},{ }_{T} C_{S}\right)$ to be indecomposable. Let $\varphi / \psi$ be an $I(U)$-minimal pair. Then $D \psi / D \varphi$ is an $I(U)^{\prime}$-minimal pair. It is realized by the pure-injective indecomposable $\operatorname{Hom}_{S}\left({ }_{R} U_{S},{ }_{T} C_{S}\right) \in I(U)^{\prime}$. By the definition of dual, $\operatorname{Hom}_{S}\left({ }_{R} U_{S},{ }_{T} C_{S}\right) \cong$ ${ }_{T} D U_{R} \cdot{ }_{R} U_{S} \cong \operatorname{Hom}_{T}\left({ }_{T} D U_{R},{ }_{T} C_{S}\right)$ is proved similarly.

Proposition 12.4. Suppose that ${ }_{R} U$ and $D U_{R}$ are both definably finitely generated and totally transcendental. Let $S \cong \operatorname{End}_{R} U$ and $T \cong \operatorname{End}_{R} D U$ and ${ }_{T} C_{S}={ }_{T} D U_{R} \otimes U_{S} \cdot{ }_{T} C_{S}$-duality is a Morita duality between $T$-mod and mod$S$ and $\left.U_{S}{ }_{T} D U\right)$ is ${ }_{T} C_{S}$-reflexive.

Proof. Both $S$ and $T$ are local rings with residue ring $T \Delta_{S}$ (cf. Proposition 6.4). By Theorem 11.2, $C_{S} \cong E\left(\Delta_{S}\right)$ and ${ }_{T} C \cong E\left({ }_{T} \Delta\right)$ so ${ }_{T} C_{S}$ is an injective cogenerator on both sides. By Theorem 10.5, both $U_{S}$ and ${ }_{T} D U$ are ${ }_{T} C_{S^{-}}$ reflexive [S, I.6.7]. By Proposition 12.3,

$$
\begin{aligned}
T & \cong \operatorname{Hom}_{R}(D U, D U) \cong \operatorname{Hom}_{R}\left(D U, \operatorname{Hom}_{S}\left({ }_{R} U_{S},{ }_{T} D U_{R} \otimes U_{S}\right)\right) \\
& \cong \operatorname{Hom}_{S}\left({ }_{T} D U_{R} \otimes U_{S},{ }_{T} D U_{R} \otimes U_{S}\right) \cong \operatorname{End}_{S} C_{S} .
\end{aligned}
$$

The proof of $S \cong \operatorname{End}_{T} C$ is similar.

Note that when Proposition 12.4 applies, both $U$ and $D U$ have a ppcomposition series.

Example 12.5. If $U$ is a finite indecomposable $R$-module, then it is pure injective and reflexive. By Theorem $6.6,|D U|=|U|$ so that $U$ and $D U$ satisfy the hypothesis of Proposition 12.4.

Example 12.6. If $R$ is representation finite, then every indecomposable (right) $R$-module is pure-injective and finitely generated [P, §11.4]. Proposition 12.4 applies to $U$ and $D U$ for every $U \in{ }_{R} I$.

Example 12.7. If $R$ is an Artin algebra with center $C(R)$, then every finitely generated module $M$ has a composition series as $C(R)$-module. Since ppdefinable subgroups of $M$ are $C(R)$-submodules, $M$ has a pp-composition series. Thus a finitely generated indecomposable $U$ is pure-injective. Let $0 \subseteq \varphi_{1}(U) \subseteq \cdots \subseteq \varphi_{n}(U)=U$ be a pp-composition series for $U$. Then for each $0 \leq k<n, \varphi_{k+1}(U) / \varphi_{k}(U) \cong C(R) \Delta$ as $C(R)$-modules so that $\Delta$ is a finitely generated $C(R)$-module. $\Delta_{C(R)}$ is also finitely generated and since $0=D \varphi_{n}(D U) \subseteq \cdots \subseteq D \varphi_{1}(D U) \subseteq D U$ is a pp-composition series for $D U$ and $D \varphi_{k}(D U) / D \varphi_{k+1}(D U) \cong \Delta_{C(R)}$ for each $0 \leq k<n, D U$ is finitely generated as $C(R)$-module and hence as $R$-module. Thus Proposition 12.4 applies to $U$ and $D U$ for every finitely generated indecomposable $U$. 


\section{REFERENCES}

[E-S] P. Eklof and G. Sabbagh, Model-completions and modules, Ann. Math. Logic 2 (1971), 251-295.

[H] Ivo Herzog, Some model theory of modules, Doctoral Dissertation, Univ. Notre Dame, 1989.

[F] C. Faith, Algebra II: Ring theory, Springer-Verlag, Berlin, Heidelberg and New York, 1976.

[P] Mike Prest, Model theory and modules, London Math. Soc. Lecture Notes Ser., vol. 130, Cambridge Univ. Press, Cambridge, 1988.

[S-E] G. Sabbagh and P. Eklof, Definability problems for modules and rings, J. Symbolic Logic 36 (1971), 623-649.

[S] Bo Stenström, Rings of quotients, Springer-Verlag, New York and Heidelberg, 1975.

[Z] M. Ziegler, Model theory of modules, Ann. Pure Appl. Logic 26 (1984), 149-213.

[ZH-Z] B. Zimmermann-Huisgen and W. Zimmermann, On the sparsity of representations of rings of pure global dimension zero, Trans. Amer. Math. Soc. 320 (1990), 695-711.

Department of Mathematics, University of California, Irvine, California 92717 Current address: Department of Mathematics, Goldsmith 216, Brandeis University, Waltham, Massachusetts 02154

E-mail address: herzog@binah.cc.brandeis.bitnet 